\title{
ITÔ FORMULA FOR MILD SOLUTIONS OF SPDES WITH GAUSSIAN AND NON-GAUSSIAN NOISE AND APPLICATIONS TO STABILITY PROPERTIES
}

\author{
S. ALBEVERIO, L. GAWARECKI, V. MANDREKAR, B. RÜDIGER, AND B. SARKAR
}

\begin{abstract}
We use Yosida approximation to find an Itô formula for mild solutions $\left\{X^{x}(t), t \geq 0\right\}$ of SPDEs with Gaussian and non-Gaussian coloured noise, the non Gaussian noise being defined through compensated Poisson random measure associated to a Lévy process. The functions to which we apply such Itô formula are in $C^{1,2}([0, T] \times H)$, as in the case considered for SDEs in 19 . Using this Itô formula we prove exponential stability and exponential ultimate boundedness properties in mean square sense for mild solutions. We also compare such Itô formula to an Itô formula for mild solutions introduced by Ichikawa in [15, and an Itô formula written in terms of the semigroup of the drift operator [6] which we extend before to the non Gaussian case.
\end{abstract}

\section{INTRODUCTION}

The Itô formula for strong solutions of SPDEs can be derived similarly as for the case of SDEs, see e.g. [13], 22] for the Gaussian case and [19], [18]. Here we derive through Yosida approximation an Itô formula for mild solutions $\left\{X^{x}(t), t \geq 0\right\}$ for SPDEs driven by a Wiener process and general Lévy processes. The functions to which we apply such Itô formula are in $C^{1,2}([0, T] \times H)$, as in the case considered for SDEs in [19]. Using this Itô formula we prove exponential stability and exponential ultimate boundedness properties in mean square sense for mild solutions. We also relate this Itô formula to an Itô formula for mild solutions of stochastic semilinear evolution equations with Gaussian noise provided by Ichikawa in [15, which we generalize to SPDEs with Lévy noise. The functions to which such Ichikawa's Itô formula can be applied are in the domain of the "weak generator" associated to the SPDE, defined in (47) below. Following Ichikawa [15] we also show in Section 4 that the Itô formula for mild solutions $\left\{X^{x}(t), t \geq 0\right\}$ induces an inequality for $\Psi(X(t))$ which can be applied to a larger set of functions $\Psi$, than those being in the domain of the "weak generator". Such inequality might also be applied to prove exponential stability of $\left\{X^{x}(t), t \geq 0\right\}$ in mean square sense, as shown through an example.

We also generalize the Itô formula obtained in [6] in terms of the semigroup of the drift operator to SPDEs with non Gaussian noise. In [6] Da Prato, Jentzen and Röckner transform the mild solution of a SPDE with Gaussian noise to a standard Itô process by using the techniques of [11, through the works of Nagy [27, [28, [29]. Different than the Itô formula derived in [6] the Itô formula obtained here by Yosida approximation allows us to study the stability and ultimate boundedness

2010 Mathematics Subject Classification. 60H15, 60G15, 60G51, 47A58, 34D05, 20Mxx.

Key words and phrases. Stochastic Partial Differential Equations, Mild solutions, Itô Formula, Generator of a Semigroup, Yosida approximation, exponential stability. 
properties of the mild solutions of SPDES (see also e.g. 20] for the non-Gaussian case and 13 for the Gaussian case.)

In Section 2. we give Preliminaries related to the existence and uniqueness of mild solutions and discuss under what assumptions the mild solution is also a strong solution. We recall how the mild solutions can be obtained in the limit by a sequence of strong solutions by using Yosida approximation techniques.

In Section 3 we recall the Itô formula for strong solutions. We then derive by Yosida approximation an Itô formula for mild solutions in Theorem 4 Section 3.3. The functions for which we compute the Itô formula are in $C^{1,2}([0, T] \times H)$, and satisfy the same conditions as those applied in [19] to strong solutions of SDEs with non Gaussian noise. In Section 3.4 we prove, using the Itô formula obtained in Theorem [4 that under suitable conditions the mild solutions of SPDEs are exponential stable (Theorem 5) and ultimate bounded (Theorem 6) in mean square sense.

In [15] Ichikawa obtained an Itô formula for mild solutions w.r.t. the Gaussian noise using their "weak generators" . This will be recalled in Section 3.5 A similar result will be derived also for the non-Gaussian noise in Theorem[7. Section 3.5. The functions for which we compute the Itô formula following Ichikawa are functions $\Psi \in C^{1,2}([0, T] \times H)$, for which the function $\mathcal{L} \Psi$, with $\mathcal{L}$ being the "weak generator" (11), can be extended to a continuous function. Ichikawa's Itô formula in Theorem 7 is compared with the Itô formula obtained in Theorem 4 in Section 3.5 through Example 2 and Example 3.

In Ichikawa's type Itô formula for mild solutions, the assumption that the function $\mathcal{L} \Psi$ can be extended to a continuous function, is rather restrictive to study the stability theory of the mild solutions. So, following Ichikawa's result for the Gaussian case, in Lemma 4 of Section 4 we assume that the function $\mathcal{L} \Psi \leq \mathcal{U}$, where $\mathcal{U}$ is a continuous function. This might be used to study the exponential stability of the mild solutions in the mean square sense (this is shown in Example 4). In Section 5 we extend Da Prato, Jentzen and Röckner's mild Itô formula to the case of non-Gaussian noise in Theorem 9 For this we used the transformation technique presented in 11. Example 3 in Section 3 can be also obtained through the Itô formula in Theorem 9

\section{PRELiminaries}

Let $K$ and $H$ be real separable Hilbert spaces. Let $(H \backslash\{0\}, \mathcal{B}(H \backslash\{0\}), \beta)$ be a $\sigma$-finite measurable space, with $\mathcal{B}(H \backslash\{0\})$ denoting the Borel sets of $H \backslash\{0\}$ and $\beta$ be a positive measure on $\mathcal{B}(H \backslash\{0\})$ with

$$
\int_{H \backslash\{0\}}\left(\|u\|_{H}^{2} \wedge 1\right) \beta(d u)<\infty .
$$

We refer to this $\beta$ as a Lévy measure on $H \backslash\{0\}$.

We shall consider a compensated Poisson random measure $(\mathrm{cPrm}) q(d s, d u):=$ $N(d s, d u)(\omega)-d s \beta(d u)$ on a filtered probability space $\left(\Omega, \mathcal{F},\left\{\mathcal{F}_{t}\right\}_{t \in[0, T]}, P\right)$ satisfying the usual hypothesis. $d s$ denotes the Lebesgue measure on $\mathcal{B}\left(\mathbb{R}_{+}\right)$and $N(d s, d u)(\omega)$ is a Poisson distributed $\sigma$-finite measure on the $\sigma$-algebra $\mathcal{B}\left(\mathbb{R}_{+}, H \backslash\{0\}\right)$, 
generated by the product semiring $\mathcal{B}\left(\mathbb{R}_{+}\right) \times \mathcal{B}(H \backslash\{0\})$ of the Borel $\sigma$-algebra $\mathcal{B}\left(\mathbb{R}_{+}\right)$ and the Borel $\sigma$-algebra $\mathcal{B}(H \backslash\{0\})$. Then $E(q(A \times B))^{2}=\beta(A) \lambda(B)$, for any $A \in \mathcal{B}(H \backslash\{0\}), B \in \mathcal{B}\left(\mathbb{R}_{+}\right), 0 \notin \bar{A},(\bar{A}$ denoting the closure of the set $A) \lambda(B)$ is the Lebesgue measure of $B$. (For more details we refer to section 1 of [1]).

The definition of stochastic integral with respect to compensated Poisson random measure and their properties are given in, e.g. [2], 18, [1, [3], [7, 23, [24, [16, [26].

We recall here some definition and known facts:

Definition 1. A family $S(t) \in \mathcal{L}(X), t \geq 0$, of bounded linear operators on a Banach space $X$ is called a strongly continuous semigroup (or a $C_{0}$-semigroup) if (S1) $S(0)=I$,

(S2) (Semigroup property) $S(t+s)=S(t) S(s)$ for every $t, s \geq 0$,

(S3) (Strong continuity property) $\lim _{t \rightarrow 0^{+}} S(t) x=x$ for every $x \in X$.

Let $S(t)$ be a $C_{0}$-semigroup on a Banach space $X$. Then there exist constants $\alpha \geq 0$ and $M \geq 1$ such that $\|S(t)\|_{\mathcal{L}(X)} \leq M e^{\alpha t}, t \geq 0$.

If $M=1$, then $S(t)$ is called a pseudo-contraction semigroup.

We consider the following stochastic partial differential equation with values in $H$,

(1)

$$
\left\{\begin{array}{r}
d X(t)=(A X(t)+F(X(t))) d t+B(X(t)) d W_{t}+\int_{H \backslash\{0\}} f(v, X(t)) q(d v, d t) ; \\
X(0)=\xi .
\end{array}\right.
$$

Where $\xi$ is an $\mathcal{F}_{0}$-measurable random variable. We assume that, the terms in (11) satisfy the following conditions:

(A1) $A$ is the infinitesimal generator of a pseudo-contraction semigroup $\{S(t), t \geq$ $0\}$ on $H$. This means in particular that there exists a constant $\alpha \in \mathbb{R}_{+}$s.t. $\|S(t)\| \leq e^{\alpha t} \cdot \mathcal{D}(A)$ denotes the domain of the linear operator $A \cdot \mathcal{D}(A)$ is dense in $H$ and $A$ is a closed linear operator.

(A2) $\left(W_{t}\right)_{t \geq 0}$ is a $K$-valued $\mathcal{F}_{t^{-}}$-Wiener process with covariance $Q$ on $\left(\Omega, \mathcal{F},\left\{\mathcal{F}_{t}\right\}_{t \in[0, T]}, P\right)$ satisfying the usual hypothesis, where $Q$ is a nonnegative definite symmetric traceclass operator on the real separable Hilbert space $K$. $\left(W_{t}\right)_{t>0}$ is assumed to be independent of the $\mathrm{cPrm} q(d v, d t)$.

(A3) $F: H \rightarrow H, B: H \rightarrow \mathcal{L}(K, H), f: H \backslash\{0\} \times H \rightarrow H$ are continuous, and Bochner measurable functions satisfying:

$$
\|F(x)\|_{H}^{2}+\operatorname{tr}\left(B(x) Q B^{*}(x)\right)+\int_{H \backslash\{0\}}\|f(v, x)\|_{H}^{2} \beta(d v) \leq l\left(1+\|x\|_{H}^{2}\right) ;
$$

and

$$
\|F(x)-F(y)\|_{H}^{2}+\operatorname{tr}\left((B(x)-B(y)) Q(B(x)-B(y))^{*}\right)
$$




$$
+\int_{H \backslash\{0\}}\|f(v, x)-f(v, y)\|_{H}^{2} \beta(d v) \leq \mathcal{K}\|x-y\|_{H}^{2} ;
$$

for all $x, y \in H$. Where $l, \mathcal{K}$ are positive constants.

Definition 2. A stochastic process $\{X(t), t \geq 0\}$ is called a mild solution of (1) in $[0, T]$

(i) $X(t)$ is $\mathcal{F}_{t}$-adapted on the filtered probability space $\left(\Omega, \mathcal{F},\left\{\mathcal{F}_{t}\right\}_{t \in[0, T]}, P\right)$,

(ii) $\{X(t), t \geq 0\}$ is jointly measurable and $\int_{0}^{T} E\left[\|X(t)\|_{H}^{2}\right] d t<\infty$, (iii)

$$
\begin{array}{r}
X(t)=S(t) \xi+\int_{0}^{t} S(t-s) F(X(s)) d s+\int_{0}^{t} S(t-s) B(X(s)) d W_{s} \\
+\int_{0}^{t} \int_{H \backslash\{0\}} S(t-s) f(v, X(s)) q(d v, d s)
\end{array}
$$

holds in $[0, T]$ a.s..

Definition 3. A stochastic process $\{X(t), t \geq 0\}$ is called a strong solution of (1) in $[0, T]$, if for all $t \leq T$

(i) $X(t)$ is $\mathcal{F}_{t}$-adapted on the filtered probability space $\left(\Omega, \mathcal{F},\left\{\mathcal{F}_{t}\right\}_{t \in[0, T]}, P\right)$,

(ii) $\{X(t), t \geq 0\}$ is càdlàg with probability one,

(iii) $X(t) \in \mathcal{D}(A), d t \otimes d P$ a.e., $\int_{0}^{T}\|A X(t)\|_{H} d t<\infty P$ a.s.,

(iv)

$$
\begin{aligned}
X(t)=\xi+\int_{0}^{t}(A X(s)+ & F(X(s))) d s+\int_{0}^{t} B(X(s)) d W_{s} \\
& +\int_{0}^{t} \int_{H \backslash\{0\}} f(v, X(s)) q(d v, d s)
\end{aligned}
$$

holds in $[0, T]$ a.s..

Let $\mathcal{L}(K, H)$ be the space of all linear bounded operators from $K$ to $H$. Let $\left\{f_{j}\right\}_{j=1}^{\infty}$ be an ONB in $K$ diagonailizing $Q$ and let the corresponding eigenvalues be $\left\{\lambda_{j}\right\}_{j=1}^{\infty}$. Let $\mathcal{L}_{2}\left(K_{Q}, H\right)$ be the space of Hilbert-Schmidt operators from $K_{Q}:=Q^{1 / 2} K$ to $H$. (see Section 2.2 of chapter 2 of [13], Chapter 4 of [7, or [21]). Let $\Lambda_{2}\left(K_{Q}, H\right)$ be a class of $\mathcal{L}_{2}\left(K_{Q}, H\right)$-valued measurable processes, $\phi(t)$ as mapping from $([0, T] \times \Omega, \mathcal{B}([0, T]) \otimes \mathcal{F})$ to $\left(\mathcal{L}_{2}\left(K_{Q}, H\right), \mathcal{B}\left(\mathcal{L}_{2}\left(K_{Q}, H\right)\right)\right)$, adapted to the filtration $\left\{\mathcal{F}_{t}\right\}_{\{t \leq T\}}$, and satisfying the condition $E\left[\int_{0}^{T}\|\phi(t)\|_{\mathcal{L}_{2}\left(K_{Q}, H\right)}^{2} d t\right]<\infty$.

Let $L_{T, \beta}^{2}(H)$ be the space s.t. $L_{T, \beta}^{2}(H):=\{\varphi:(H \backslash\{0\}) \times[0, T] \times \Omega \rightarrow H$, such that $\varphi$ is jointly measurable and $\mathcal{F}_{t}$-adapted for all $v \in H \backslash\{0\}, t \in[0, T]$ with $\left.E\left[\int_{0}^{T} \int_{H \backslash\{0\}}\|\varphi(v, t)\|_{H}^{2} \beta(d v) d t\right]<\infty\right\}$.

Let us assume in the next Lemmma that (A1), (A2) hold.

Lemma 1. a) Let $\tilde{B}(s) \in \Lambda_{2}\left(K_{Q}, H\right)$ with $E\left[\int_{0}^{T}\|\tilde{B}(s)\|_{\mathcal{L}_{2}\left(K_{Q}, H\right)}^{2} d s\right]<\infty$. Then for any stopping time $\tau$, there exists a constant $C_{1}$, depending on $\alpha, T$ s.t.

$$
E\left[\sup _{0 \leq t \leq T \wedge \tau}\left\|\int_{0}^{t} S(t-s) \tilde{B}(s) d W_{s}\right\|_{H}^{2}\right] \leq C_{1} E\left[\int_{0}^{T \wedge \tau}\|\tilde{B}(s)\|_{\mathcal{L}_{2}\left(K_{Q}, H\right)}^{2} d s\right] .
$$


b) Let $\varphi \in L_{T, \beta}^{2}(H)$ and $\tau$ be a stopping time. Then, there exists a constant $C_{2}$, depending on $\alpha, T$ s.t.

$$
\begin{array}{r}
E\left[\sup _{0 \leq t \leq T \wedge \tau}\left\|\int_{0}^{t} \int_{H \backslash\{0\}} S(t-s) \varphi(v, s) q(d v, d s)\right\|_{H}^{2}\right] \\
\leq C_{2} E\left[\int_{0}^{T \wedge \tau} \int_{H \backslash\{0\}}\|\varphi(v, s)\|_{H}^{2} \beta(d v) d s\right] .
\end{array}
$$

Proof. For the proof of the first inequality we refer Lemma 3.3(b) of [13]. For the proof of the second inequality we refer Lemma 5.1.9(1) of [18], [4].

Moreover, let us assume in this section from this point on that (A1), (A2), (A3) holds.

Let $\left\{\xi(t), t \in[0, T]\right.$ be a $\mathcal{F}_{t^{-}}$-adapted process for all $t \in[0, T]$.

Let us define

$$
\begin{aligned}
I(t, \xi(t)) & =\int_{0}^{t} S(t-s) F(\xi(s)) d s+\int_{0}^{t} S(t-s) B(\xi(s)) d W_{s} \\
& +\int_{0}^{t} \int_{H \backslash\{0\}} S(t-s) f(v, \xi(s)) q(d v, d s), \quad t \in[0, T] .
\end{aligned}
$$

Lemma 2. Let $E\left[\sup _{0 \leq s \leq T}\|\xi(s)\|_{H}^{2}\right]<\infty$. Then for any stopping time $\tau$

$$
E\left[\sup _{0 \leq s \leq t \wedge \tau}\|I(s, \xi(s))\|_{H}^{2}\right] \leq C_{3}\left(t+\int_{0}^{t} E\left[\sup _{0 \leq u \leq s \wedge \tau}\|\xi(u)\|_{H}^{2}\right] d s\right),
$$

where $C_{3}$ is a constant depending on $\alpha, T$ and $l$.

Proof. For the proof we refer Lemma 3.4 of Chapter 3 of [13] and Theorem 5.2.1 of [18. In [13] it is done for the Gaussian case and in [18] it is done for the nonGaussian case.

Lemma 3. Let $E\left[\sup _{0 \leq s \leq T}\|\xi(s)\|_{H}^{2}\right]<\infty$. Then

$$
E\left[\sup _{0 \leq s \leq t}\left\|I\left(s, \xi_{1}(s)\right)-I\left(s, \xi_{2}(s)\right)\right\|_{H}^{2}\right] \leq C_{4} \int_{0}^{t} E\left[\sup _{0 \leq u \leq s}\left\|\xi_{1}(u)-\xi_{2}(u)\right\|_{H}^{2}\right] d s,
$$

where $C_{4}$ is a constant depending on $\alpha, T$ and $\mathcal{K}$.

Proof. For the proof we refer Lemma 3.5 of [13] and Lemma 5.2.2 of [18]. In [13] it is done for the Gaussian case and in [18 it is done for the non-Gaussian case.

2.1. Existence and uniqueness of the mild solutions. Let $(D[0, T], H)$ be the space of càdlàg functions defined on $[0, T]$ and with values in $H$, with the sup norm $\|\cdot\|_{\infty}:=\sup _{t \in[0, T]}\|\cdot\|_{H}$. Let $\mathcal{H}_{2}^{T}$ denote the space of $(D[0, T], H)$-valued random processes $\xi(t)$, which are jointly measurable, adapted to the filtration $\left\{\mathcal{F}_{t}\right\}_{t \in[0, T]}$, with $E\left[\sup _{0 \leq s \leq T}\|\xi(s)\|_{H}^{2}\right]<\infty$. The space $\mathcal{H}_{2}^{T}$, equipped with the norm $\|X\|_{\mathcal{H}_{2}^{T}}:=$ $\left(E\left[\sup _{0 \leq s \leq T}\|X(s)\|_{H}^{2}\right]\right)^{1 / 2}$ is a Banach space (see Section 4.1 of [18]).

Theorem 1. Let $\{S(t), t \geq 0\}$ be a pseudo-contraction semigroup generated by $A$ satisfying assumption (A1). Suppose assumption (A2) holds. Let the coefficients $F, B, f$ satisfy assumption (A3), and assume that $E\left[\|X(0)\|_{H}^{2}\right]<\infty$. Then for 
each $T>0$ equation (1) has a unique mild solution $X^{\xi} \in(D[0, T], H)$ satisfying $E\left[\sup _{0 \leq s \leq T}\left\|X^{\xi}(s)\right\|_{H}^{2}\right]<\infty$, i.e. the mild solution is in $\mathcal{H}_{2}^{T}$.

Proof. For the proof we refer Theorem 3.3 of [13] and Theorem 5.2.3 of [18. In [13] it is done for the Gaussian case and in $[18$ it is done for the non-Gaussian case. The proofs use Lemma 1 and Lemma 3, In Lemma 1 the condition $\tilde{B}(s) \in \Lambda_{2}\left(K_{Q}, H\right)$, for $\tilde{B}(s):=B(X(s))$ is a consequence of assumption (A3).

\subsection{When a mild solution is a strong solution.}

Theorem 2. Let $\{S(t), t \geq 0\}$ be a pseudo-contraction semigroup generated by $A$ satisfying assumption (A1). Suppose assumption (A2) holds. Let the coefficients $F, B, f$ satisfy assumption (A3), and assume that $E\left[\|X(0)\|_{H}^{2}\right]<\infty$.

Suppose $\xi \in \mathcal{D}(A)$ a.s., $S(t-r) F(y) \in \mathcal{D}(A), S(t-r) B(y) \in \mathcal{D}(A), S(t-r) f(v, y) \in$ $\mathcal{D}(A)$; for all $r<t, y \in H$ and $v \in H \backslash\{0\}$.

Then for any $T>0$ the mild solution $\{X(t)\}_{t \in[0, T]}$ of (1) satisfies $X(t) \in \mathcal{D}(A)$ $d t \otimes d P$ a.e., and is a strong solution.

Proof. Here we follow exactly the proof of Proposition 2.3 of [14 and Theorem 3.2 of [20. In [14] the Gaussian case is considered and in [20] the non-Gaussian case is considered.

From the assumptions it follows

$$
\begin{gathered}
\int_{0}^{t} A X(s) d s=\int_{0}^{t} A S(s) \xi d s+\int_{0}^{t} \int_{0}^{s} A S(s-r) F(X(r)) d r d s+\int_{0}^{t} \int_{0}^{s} A S(s-r) B(X(r)) d W_{r} d s \\
+\int_{0}^{t} \int_{0}^{s} \int_{H \backslash\{0\}} A S(s-r) f(v, X(r)) q(d v, d r) d s,
\end{gathered}
$$

since, we know

$$
\begin{aligned}
\int_{0}^{t} \int_{0}^{s} f(s-r) g(r) d r d s & =\int_{0}^{t} \int_{0}^{t} f(s-r) g(r) \chi_{[0, s]}(r) d r d s \\
& =\int_{0}^{t} \int_{0}^{t} f(s-r) g(r) \chi_{[0, s]}(r) d s d r \\
& =\int_{0}^{t} \int_{0}^{t} f(s-r) g(r) \chi_{[r, t]}(s) d s d r \\
& =\int_{0}^{t} \int_{r}^{t} f(s-r) g(r) d s d r
\end{aligned}
$$

and, by the given conditions we have

$$
\int_{0}^{T} \int_{0}^{t}\|A S(t-r) F(X(r))\| d r d t<\infty
$$

with probability one,

$$
\int_{0}^{T} \int_{0}^{t}\|A S(t-r) B(X(r))\|^{2} d r d t<\infty
$$


with probability one and

$$
\int_{0}^{T} \int_{0}^{t} \int_{H \backslash\{0\}} E\|A S(t-r) f(v, X(r))\|^{2} \beta(d v) d r d t<\infty
$$

with probability one. Hence, by applying Fubini theorem we get,

$$
\begin{aligned}
\int_{0}^{t} A X(s) d s= & \int_{0}^{t} A S(s) \xi d s+\int_{0}^{t} \int_{r}^{t} A S(s-r) F(X(r)) d s d r+\int_{0}^{t} \int_{r}^{t} A S(s-r) B(X(r)) d s d W_{r} \\
& +\int_{0}^{t} \int_{H \backslash\{0\}} \int_{r}^{t} A S(s-r) f(v, X(r)) d s q(d v, d r) .
\end{aligned}
$$

(For the stochastic Fubini theorem we refer theorem (2.8) of [13] and Appendix A of [18]). Now we apply the formula,

$$
\int_{0}^{t} A S(s) \xi d s=S(t) \xi-\xi
$$

when $\xi \in \mathcal{D}(A)$ a.s.. Hence $A X(t)$ is integrable with probability one and

$$
\begin{gathered}
\int_{0}^{t} A X(s) d s=S(t) \xi-\xi+\int_{0}^{t} S(t-r) F(X(r)) d r-\int_{0}^{t} F(X(r)) d r \\
+\int_{0}^{t} S(t-r) B(X(r)) d W_{r}-\int_{0}^{t} B(X(r)) d W_{r} \\
+\int_{0}^{t} \int_{H \backslash\{0\}} S(t-r) f(v, X(r)) q(d v, d r)-\int_{0}^{t} \int_{H \backslash\{0\}} f(v, X(r)) q(d v, d r) .
\end{gathered}
$$

Hence

$$
\begin{aligned}
\int_{0}^{t} A X(r) d r & =X(t)-\xi-\int_{0}^{t} F(X(r)) d r-\int_{0}^{t} B(X(r)) d W_{r} \\
& -\int_{0}^{t} \int_{H \backslash\{0\}} f(v, X(r)) q(d v, d r) .
\end{aligned}
$$

Therefore

$$
\begin{gathered}
X(t)=\xi+\int_{0}^{t} A X(r) d r+\int_{0}^{t} F(X(r)) d r+\int_{0}^{t} B(X(r)) d W_{r} \\
+\int_{0}^{t} \int_{H \backslash\{0\}} f(v, X(r)) q(d v, d r) .
\end{gathered}
$$

By Definition [3, $\{X(t), t \in[0, T]\}$ is a strong solution of equation (11). 
2.3. Approximating a mild solution by the strong solutions. We assume that the assumptions (A1), (A2), (A3) are satisfied for equation (11). Let us consider the approximating system of equation (11),

$d X(t)=\left(A X(t)+R_{n} F(X(t))\right) d t+R_{n} B(X(t)) d W_{t}+\int_{H \backslash\{0\}} R_{n} f(v, X(t)) q(d v, d t)$

$$
X(0)=\xi \in \mathcal{D}(A) a . s . .
$$

where $R_{n}=n R(n, A)$, and $R(n, A)=(n I-A)^{-1}$ denotes the resolvent of $A$ evaluted at $n \in \mathbb{N}$, with $n \in \rho(A), \rho(A)$ denoting the resolvent set of $A$. We have $R_{n}: H \rightarrow \mathcal{D}(A)$ and $A_{n}=A R_{n}$ are the Yosida approximations of $A$ (see Chapter 1 of [13]).

By applying Theorem 1. we conclude that equation (3) has a unique mild solution, denoted by $X_{n}^{\xi}(t)$. Then

$$
\begin{aligned}
X_{n}^{\xi}(t)=S(t) \xi & +\int_{0}^{t} S(t-s) R_{n} F\left(X_{n}^{\xi}(s)\right) d s+\int_{0}^{t} S(t-s) R_{n} B\left(X_{n}^{\xi}(s)\right) d W_{s} \\
& +\int_{0}^{t} \int_{H \backslash\{0\}} S(t-s) R_{n} f\left(v, X_{n}^{\xi}(s)\right) q(d v, d s) .
\end{aligned}
$$

Since the range $\mathcal{R}(R(n, A)) \subset \mathcal{D}(A)$ (see Chapter 1 of [13]) and the conditions of Theorem 2 are satisfied, we conclude that $X_{n}^{\xi}(t) \in \mathcal{D}(A)$ is also a strong solution.

Now we are in a position to approximate the mild solution of equation (1) by the strong solutions of equation (3). The mild solution of equation (11), say $X^{\xi}(t)$, satisfies by definition

$$
\begin{aligned}
X^{\xi}(t)=S(t) \xi & +\int_{0}^{t} S(t-s) F\left(X^{\xi}(s)\right) d s+\int_{0}^{t} S(t-s) B\left(X^{\xi}(s)\right) d W_{s} \\
& +\int_{0}^{t} \int_{H \backslash\{0\}} S(t-s) f\left(v, X^{\xi}(s)\right) q(d v, d s) .
\end{aligned}
$$

Theorem 3. The stochastic partial differential equation (3) has a unique strong solution $\left\{X_{n}^{\xi}(t), t \geq 0\right\}$ in $D\left([0, T], L_{2}((\Omega, \mathcal{F}, P), H)\right.$ for $T$ finite. Moreover

$$
\lim _{n \rightarrow \infty} E\left[\sup _{0 \leq t \leq T}\left\|X_{n}^{\xi}(t)-X^{\xi}(t)\right\|_{H}^{2}\right]=0
$$

where $\left\{X^{\xi}(t), t \geq 0\right\}$ is the mild solution of equation (1).

Proof. In Theorem 1 we have already proved that there exists a unique solution of (3) in $D\left([0, T], L_{2}((\Omega, \mathcal{F}, P), H)\right.$ and by Theorem 2 this is also a strong solution. Now we will prove (6). We have

$$
E\left[\sup _{0 \leq t \leq T}\left\|X_{n}^{\xi}(t)-X^{\xi}(t)\right\|_{H}^{2}\right]
$$




$$
\begin{aligned}
= & E\left[\sup _{0 \leq t \leq T} \| \int_{0}^{t} S(t-s)\left(R_{n} F\left(X_{n}^{\xi}(s)\right)-F\left(X^{\xi}(s)\right)\right) d s\right. \\
& +\int_{0}^{t} S(t-s)\left(R_{n} B\left(X_{n}^{\xi}(s)\right)-B\left(X^{\xi}(s)\right)\right) d W_{s} \\
& \left.+\int_{0}^{t} \int_{H \backslash\{0\}} S(t-s)\left(R_{n} f\left(v, X_{n}^{\xi}(s)\right)-f\left(v, X^{\xi}(s)\right)\right) q(d v, d s) \|_{H}^{2}\right] \\
\leq & C\left\{E\left[\sup _{0 \leq s \leq t}\left\|\int_{0}^{s} S(s-r) R_{n}\left(F\left(X_{n}^{\xi}(r)\right)-F\left(X^{\xi}(r)\right)\right) d r\right\|_{H}^{2}\right]\right. \\
+ & E\left[\sup _{0 \leq s \leq t}\left\|\int_{0}^{s} S(s-r)\left(R_{n}-I\right) F\left(X^{\xi}(r)\right) d r\right\|_{H}^{2}\right] \\
+ & E\left[\sup _{0 \leq s \leq t}\left\|\int_{0}^{s} S(s-r) R_{n}\left(B\left(X_{n}^{\xi}(r)\right)-B\left(X^{\xi}(r)\right)\right) d W_{r}\right\|_{H}^{2}\right] \\
+ & C_{1} E\left[\int_{0}^{t}\left\|\left(R_{n}-I\right) B\left(X^{\xi}(r)\right)\right\|_{\mathcal{L}_{2}\left(K_{Q}, H\right)}^{2} d r\right] \\
+ & E\left[\sup _{0 \leq s \leq t}\left\|\int_{0}^{s} \int_{H \backslash\{0\}} S(s-r) R_{n}\left(f\left(v, X_{n}^{\xi}(r)\right)-f\left(v, X^{\xi}(r)\right)\right) q(d v, d r)\right\|_{H}^{2}\right] \\
+ & \left.C_{2} E\left[\int_{0}^{t} \int_{H \backslash\{0\}}\left\|\left(R_{n}-I\right) f\left(v, X^{\xi}(r)\right)\right\|_{H}^{2} \beta(d v) d r\right]\right\},
\end{aligned}
$$

where $C, C_{1}, C_{2}$ are constants depending on $\alpha$ and $T$. By Lemma 3 the first, third and fifth summands are bounded by $\mathcal{G}_{1} \mathcal{K} \int_{0}^{t} E\left[\sup _{0 \leq r \leq s}\left\|X_{n}^{\xi}(r)-X^{\xi}(r)\right\|_{H}^{2}\right] d r$, for $n>n_{0}$ ( $n_{0}$ sufficiently large), where $\mathcal{G}_{1}$ is a constant which depends on $\sup _{0 \leq t \leq T}\|S(t)\|_{\mathcal{L}(H)}$ and $\sup _{n>n_{0}}\left\|R_{n}\right\|_{\mathcal{L}(H)}$ and $\mathcal{K}$ is the Lipschitz constant in (A3).

By the properties of $R_{n}$, the integrands in the second, fourth and sixth summands converge to zero, as $n \rightarrow \infty$. The integrands are bounded by $\mathcal{G}_{2} l\left(1+\left\|X^{\xi}(r)\right\|_{H}^{2}\right)$ (by condition (A3)) for some constant $\mathcal{G}_{2}$ depending on $\|S(t)\|_{\mathcal{L}(H)}$ and $\left\|R_{n}\right\|_{\mathcal{L}(H)}$, and the constant $l$ is the linear growth condition appearing in (A3). So by Lebesgue Dominated Convergence Theorem the integrals converge to zero as $n \rightarrow \infty$. Therefore there exists $\epsilon>0$ s.t. for sufficiently large $n$ each of the three summands are less or equal $\epsilon$. So for sufficiently large $n$,

$$
E\left[\sup _{0 \leq t \leq T}\left\|X_{n}^{\xi}(t)-X^{\xi}(t)\right\|_{H}^{2}\right] \leq 3 \mathcal{G}_{1} \mathcal{K} \int_{0}^{t} E\left[\sup _{0 \leq r \leq s}\left\|X_{n}^{\xi}(r)-X^{\xi}(r)\right\|_{H}^{2}\right] d r+3 \epsilon .
$$

By Gronwall's lemma (for sufficiently large $n$ ),

$$
E\left[\sup _{0 \leq t \leq T}\left\|X_{n}^{\xi}(t)-X^{\xi}(t)\right\|_{H}^{2}\right] \leq 3 \epsilon e^{3 \mathcal{G}_{1} \mathcal{K} t} .
$$

From this we conclude that

$$
\lim _{n \rightarrow \infty} E\left[\sup _{0 \leq t \leq T}\left\|X_{n}^{\xi}(t)-X^{\xi}(t)\right\|_{H}^{2}\right]=0 .
$$

We call $\left\{X_{n}^{\xi}(t)\right\}$ in the above theorem the Yosida approximation of the mild solution of (11). 


\section{ITÔ FORMULA FOR STRONG AND MILD SOLUTIONS AND APPLICATIONS}

In this Section (including all subsections) we assume that the assumptions (A1), (A2), (A3) are satisfied for equation (1).

3.1. Itô formula for strong solutions. Let all the assumption in Theorem 1 be satisfied, so that a unique mild solution of (1) exists in $\mathcal{H}_{2}^{T}$ for all $T>0$. Let us assume here that $\xi \in \mathcal{D}(A)$ a.s. and also that $\left\{X^{\xi}(t), t \geq 0\right\}$ is a strong solution of (1). Under these assumptions the Itô formula known for solutions of Banach valued stochastic differential equations with Gaussian and Lévy noise hold also for the strong solution $\left\{X^{\xi}(t), t \geq 0\right\}$ of (11). We recall this result here in the more general statement obtained in [19] (see Remark 2 for the more known statement of Itô formula). We need to recall some notation and definition:

Let $C^{1,2}([0, T] \times H)\left(C_{b}^{1,2}([0, T] \times H)\right)$ denote the class of real valued continuous functions $\Psi$ on $[0, T] \times H$ with continuous (uniformly bounded) Fréchet derivatives $\partial_{s} \Psi(s, x), \partial_{x} \Psi(s, x), \partial_{s} \partial_{x} \Psi(s, x), \partial_{x} \partial_{s} \Psi(s, x)$ and $\partial_{x} \partial_{x} \Psi(s, x)$.

Definition 4. We call a continuous, non-decreasing function $h: \mathbb{R}_{+} \rightarrow \mathbb{R}_{+}$quasisublinear if there is a constant $C>0$ such that

$$
\begin{gathered}
h(x+y) \leq C(h(x)+h(y)), \quad x, y \in \mathbb{R}_{+}, \\
h(x y) \leq C h(x) h(y), \quad x, y \in \mathbb{R}_{+} .
\end{gathered}
$$

Let $\Psi \in C^{1,2}([0, T] \times H)$. Assume moreover that the following conditions hold:

$$
\left\|\Psi_{x}(s, x)\right\|_{H} \leq h_{1}\left(\|x\|_{H}\right)
$$

and

$$
\left\|\Psi_{x x}(s, x)\right\|_{\mathcal{L}(H)} \leq h_{2}\left(\|x\|_{H}\right) .
$$

for $h_{1}, h_{2}: \mathbb{R}_{+} \rightarrow \mathbb{R}_{+}$quasi-sublinear functions such that

$$
\begin{array}{r}
\int_{0}^{T} \int_{H \backslash\{0\}}\|f(v, X(s))\|^{2} \beta(d v) d s+\int_{0}^{T} \int_{H \backslash\{0\}} h_{1}(\|f(v, X(s))\|)^{2}\|f(v, X(s))\|^{2} \beta(d v) d s \\
+\int_{0}^{T} \int_{H \backslash\{0\}} h_{2}(\|f(v, X(s))\|)\|f(v, X(s))\|^{2} \beta(d v) d s<\infty
\end{array}
$$

$P$-a.s., for all $T>0$. Then the following usual Itô formula holds:

$$
\begin{aligned}
& \Psi\left(t, X^{\xi}(t)\right)-\Psi(0, \xi)=\int_{0}^{t}\left(\Psi_{s}\left(s, X^{\xi}(s)\right)+\mathcal{L} \Psi\left(s, X^{\xi}(s)\right)\right) d s \\
& +\int_{0}^{t}\left\langle\Psi_{x}\left(s, X^{\xi}(s)\right), B\left(X^{\xi}(s)\right) d W_{s}\right\rangle_{H} \\
& +\int_{0}^{t} \int_{H \backslash\{0\}}\left[\Psi\left(s, X^{\xi}(s)+f\left(v, X^{\xi}(s)\right)\right)-\Psi\left(s, X^{\xi}(s)\right)\right] q(d v, d s) .
\end{aligned}
$$


with

$\mathcal{L} \Psi(s, x):=\left\langle\Psi_{x}(s, x), A x+F(x)\right\rangle_{H}+\frac{1}{2} \operatorname{tr}\left(\Psi_{x x}(s, x)(B(x)) Q(B(x))^{*}\right)$

$$
+\int_{H \backslash\{0\}}\left[\Psi(s, x+f(v, x))-\Psi(s, x)-\left\langle\Psi_{x}(s, x), f(v, x)\right\rangle_{H}\right] \beta(d v) \quad s \in \mathbb{R}, x \in \mathcal{D}(A) .
$$

This follows from the Itô formula for strong solutions of SDEs with Lévy noise analysed in [19] and further in Theorem 3.7.2 in [18] (for the more general case of non deterministic coefficients, like e.g. assumed here in Theorem 10.

Remark 1. A sufficient condition for (9) to hold is

$$
\begin{array}{r}
\int_{H \backslash\{0\}}\|f(v, x)\|^{2} \beta(d v)+\int_{H \backslash\{0\}}\left(h_{1}(\|f(v, x)\|)\right)^{2}\|f(v, x)\|^{2} \beta(d v) \\
\left.+\int_{H \backslash\{0\}} h_{2}(\|f(v, x)\|) \| f(v, x)\right) \|^{2} \beta(d v)<\infty \quad \forall x \in H,
\end{array}
$$

as we assume the hypothesis in Theorem[1] to be satisfied, so that $E\left[\sup _{0 \leq s \leq T}\left\|X^{\xi}(s)\right\|_{H}^{2}\right]<$ $\infty$.

Remark 2. if $\Psi \in C_{b}^{1,2}([0, T] \times H)$ then the assumptions (77), (8) and (9) can be skipped, as we are in the case considered in [24], [16]. In fact for inequality (77), (8), and (9) we can choose the quasi linear functions $h_{1}$ and $h_{2}$ to be constants. In particular (9) holds thanks to the assumption (A3) (see the first Example at page 184 in [19]).

3.2. Itô formula for Yosida approximations of mild solutions. Let all the assumption in Theorem 1 be satisfied and $\xi \in \mathcal{D}(A)$ a.s.. Let $\left\{X_{n}^{\xi}(t)\right\}$ be the Yosida approximation of the mild solution of (1). Assume that the quasi-linear functions satisfy (7), (8) and (9). Then due to Theorem 3 and Theorem 3.7.2 in [18 the following usual Itô formula holds:

$$
\begin{aligned}
& \Psi\left(t, X_{n}^{\xi}(t)\right)-\Psi(0, \xi)=\int_{0}^{t}\left(\Psi_{s}\left(s, X_{n}^{\xi}(s)\right)+\mathcal{L}_{n} \Psi\left(s, X_{n}^{\xi}(s)\right)\right) d s \\
& +\int_{0}^{t}\left\langle\Psi_{x}\left(s, X_{n}^{\xi}(s)\right), R_{n} B\left(X_{n}^{\xi}(s)\right) d W_{s}\right\rangle_{H} \\
& +\int_{0}^{t} \int_{H \backslash\{0\}}\left[\Psi\left(s, X_{n}^{\xi}(s)+R_{n} f\left(v, X_{n}^{\xi}(s)\right)\right)-\Psi\left(s, X_{n}^{\xi}(s)\right)\right] q(d v, d s),
\end{aligned}
$$

where

$$
\begin{aligned}
& \mathcal{L}_{n} \Psi(s, x)=\left\langle\Psi_{x}(s, x), A x+R_{n} F\left(X_{n}^{\xi}(s)\right)\right\rangle_{H} \\
& +\frac{1}{2} \operatorname{tr}\left(\Psi_{x x}(s, x)\left(R_{n} B\left(X_{n}^{\xi}(s)\right)\right) Q\left(R_{n} B\left(X_{n}^{\xi}(s)\right)\right)^{*}\right) \\
& +\int_{H \backslash\{0\}}\left[\Psi\left(s, x+R_{n} f(v, x)-\Psi(s, x)-\left\langle\Psi_{x}(s, x), R_{n} f(v, x)\right\rangle_{H}\right] \beta(d v) \quad s \in \mathbb{R}, x \in \mathcal{D}(A) .\right.
\end{aligned}
$$

Remark 3. If $\Psi \in C_{b}^{1,2}([0, T] \times H)$, then (7), (8) and (9) can be ignored, the Itô formula (13) still holding (See Remark Q) 
3.3. An Itô formula for mild solutions obtained through Yosida approximation. By using Yosida approximations we will derive an Itô formula for mild solutions of (11) which are not necessarily strong solutions.

Theorem 4. Let $\{S(t), t \geq 0\}$ be a pseudo-contraction semigroup generated by $A$ satisfying assumption (A1). Suppose moreover that assumption (A2) holds. Let the coefficients $F, B, f$ satisfy assumption (A3), and assume that $\xi \in \mathcal{D}(A)$ a.s.. Moreover assume that (7), (8), (9) hold.

Then the following Itô Formula for the mild solution $X^{\xi}$ of equation (11) holds $P$-a.s. for all $t \in[0, T]$

$$
\begin{aligned}
& \lim _{n \rightarrow \infty} \int_{0}^{t}\left\langle\Psi_{x}\left(s, X_{n}^{\xi}(s)\right), A X_{n}^{\xi}(s)\right\rangle_{H} d s \\
& =\Psi\left(t, X^{\xi}(t)\right)-\Psi(0, \xi)-\int_{0}^{t}\left(\Psi_{s}\left(s, X^{\xi}(s)\right)\right) d s-\int_{0}^{t}\left\langle\Psi_{x}\left(s, X^{\xi}(s)\right), F\left(X^{\xi}(s)\right)\right\rangle_{H} d s \\
& -\int_{0}^{t} \frac{1}{2} \operatorname{tr}\left(\Psi_{x x}\left(s, X^{\xi}(s)\right)\left(B\left(X^{\xi}(s)\right)\right) Q\left(B\left(X^{\xi}(s)\right)\right)^{*}\right) d s \\
& -\int_{0}^{t} \int_{H \backslash\{0\}}\left[\Psi\left(s, X^{\xi}(s)+f\left(v, X^{\xi}(s)\right)\right)-\Psi\left(s, X^{\xi}(s)\right)-\left\langle\Psi_{x}\left(s, X^{\xi}(s)\right), f\left(v, X^{\xi}(s)\right)\right\rangle_{H}\right] \beta(d v) d s \\
& -\int_{0}^{t}\left\langle\Psi_{x}\left(s, X^{\xi}(s)\right), B\left(X^{\xi}(s)\right) d W_{s}\right\rangle_{H} \\
& -\int_{0}^{t} \int_{H \backslash\{0\}}\left[\Psi\left(s, X^{\xi}(s)+f\left(v, X^{\xi}(s)\right)\right)-\Psi\left(s, X^{\xi}(s)\right)\right] q(d v, d s) .
\end{aligned}
$$

We will use in the proof of Theorem 4 below the Itô formula for the Yosida approximations of the mild solution $X^{\xi}$ of equation (11). It is then easy checked that due Remark 3 the following Corollary is a direct consequence of the proof of Theorem 4

Corollary 1. Let $\{S(t), t \geq 0\}$ be a pseudo-contraction semigroup generated by $A$ satisfying assumption (A1). Suppose assumption (A2) holds. Let the coefficients $F, B, f$ satisfy assumption (A3), and assume that $\xi \in \mathcal{D}(A)$ a.s.. Then for each $\Psi \in C_{b}^{1,2}([0, T] \times H)$ the Itô Formula (15) holds

Here we will prove Theorem 4

Proof. From (13),

$$
\begin{aligned}
& \Psi\left(t, X_{n}^{\xi}(t)\right)-\Psi(0, \xi) \\
& =\int_{0}^{t}\left(\Psi_{s}\left(s, X_{n}^{\xi}(s)\right)+\mathcal{L}_{n} \Psi\left(s, X_{n}^{\xi}(s)\right)\right) d s+\int_{0}^{t}\left\langle\Psi_{x}\left(s, X_{n}^{\xi}(s)\right), R_{n} B\left(X_{n}^{\xi}(s)\right) d W_{s}\right\rangle_{H} \\
& +\int_{0}^{t} \int_{H \backslash\{0\}}\left[\Psi\left(s, X_{n}^{\xi}(s)+R_{n} f\left(x, X_{n}^{\xi}(s)\right)\right)-\Psi\left(s, X_{n}^{\xi}(s)\right)\right] q(d x, d s) .
\end{aligned}
$$


Now we substite $\mathcal{L}_{n}\left(\Psi\left(s, X_{n}^{\xi}(s)\right)\right)$,

$$
\begin{aligned}
= & \int_{0}^{t}\left(\Psi_{s}\left(s, X_{n}^{\xi}(s)\right)\right) d s+\int_{0}^{t}\left\langle\Psi_{x}\left(s, X_{n}^{\xi}(s)\right), A X_{n}^{\xi}(s)+R_{n} F\left(X_{n}^{\xi}(s)\right)\right\rangle_{H} d s \\
& +\int_{0}^{t} \frac{1}{2} \operatorname{tr}\left(\Psi_{x x}\left(s, X_{n}^{\xi}(s)\right)\left(R_{n} B\left(X_{n}^{\xi}(s)\right)\right) Q\left(R_{n} B\left(X_{n}^{\xi}(s)\right)\right)^{*}\right) d s \\
& +\int_{0}^{t} \int_{H \backslash\{0\}}\left[\Psi\left(s, X_{n}^{\xi}(s)+R_{n} f\left(v, X_{n}^{\xi}(s)\right)\right)-\Psi\left(s, X_{n}^{\xi}(s)\right)-\left\langle\Psi_{x}\left(s, X_{n}^{\xi}(s)\right), R_{n} f\left(v, X_{n}^{\xi}(s)\right)\right\rangle_{H}\right] \beta(d v) d s \\
& +\int_{0}^{t}\left\langle\Psi_{x}\left(s, X_{n}^{\xi}(s)\right), R_{n} B\left(X_{n}^{\xi}(s)\right) d W_{s}\right\rangle_{H} \\
& +\int_{0}^{t} \int_{H \backslash\{0\}}\left[\Psi\left(s, X_{n}^{\xi}(s)+R_{n} f\left(v, X_{n}^{\xi}(s)\right)\right)-\Psi\left(s, X_{n}^{\xi}(s)\right)\right] q(d v, d s) .
\end{aligned}
$$

Now our task is to show that the above equation converges $P$-a.s.(term by term) and also to find the limit.

The convergence in Theorem 3 (equation ([6]) allows us to choose a subsequence $X_{n_{k}}^{\xi}$ such that,

$$
X_{n_{k}}^{\xi}(t) \rightarrow X^{\xi}(t), 0 \leq t \leq T, P \text {-a.s. }
$$

We will denote such a subsequence again by $X_{n}^{\xi}$.

In fact, we can say that

$$
\sup _{0 \leq t \leq T}\left\|X_{n}(t)-X(t)\right\|_{H} \rightarrow 0
$$

$P$ a.s.. This implies that the set

$$
S=\left\{X_{n}(t), X(t): n=1,2 \ldots, 0 \leq t \leq T\right\}
$$

is bounded in $H$, hence all the values of $\Psi$ and its derivatives evaluated on $S$ are bounded by some constant. Now we are ready to show the term by term convergence of equation (16).

First consider the first term of the L.H.S. of eq. (16). Since $\Psi$ is continuous, from (17) we conclude that

$$
\Psi\left(t, X_{n}^{\xi}(t)\right) \rightarrow \Psi\left(t, X^{\xi}(t)\right),
$$

$P$-a.s.

Now consider the first term of the R.H.S. of eq. (16). $\Psi_{s}$ is continuous, $\Psi_{s}\left(s, X_{n}^{\xi}(s)\right)<C$ by equation (17). So by applying Lebesgue dominated convergence Theorem we conclude that

$$
\int_{0}^{t}\left(\Psi_{s}\left(s, X_{n}^{\xi}(s)\right)\right) d s \rightarrow \int_{0}^{t}\left(\Psi_{s}\left(s, X^{\xi}(s)\right)\right) d s
$$

P-a.s.

Now consider the second term of the R.H.S. of eq. (16), 


$$
\begin{aligned}
& \int_{0}^{t}\left\langle\Psi_{x}\left(s, X_{n}^{\xi}(s)\right), A X_{n}^{\xi}(s)+R_{n} F\left(X_{n}^{\xi}(s)\right)\right\rangle_{H} d s \\
& =\int_{0}^{t}\left\langle\Psi_{x}\left(s, X_{n}^{\xi}(s)\right), A X_{n}^{\xi}(s)\right\rangle_{H} d s+\int_{0}^{t}\left\langle\Psi_{x}\left(s, X_{n}^{\xi}(s)\right), R_{n} F\left(X_{n}^{\xi}(s)\right)\right\rangle_{H} d s .
\end{aligned}
$$

Since $\Psi_{x}$ is continuous, by (17) we get $\Psi_{x}\left(s, X_{n}^{\xi}(s)\right) \rightarrow \Psi_{x}\left(s, X^{\xi}(s)\right)$. Since $F$ is continuous and $R_{n}\left(F\left(X_{n}^{\xi}(s)\right)\right.$ is a double sequence, therefore we have

$$
\begin{aligned}
\left\|R_{n}\left(F\left(X_{n}(s)\right)\right)-F(X(s))\right\|_{H} & \leq\left\|R_{n}\left(F\left(X_{n}(s)\right)-F(X(s))\right)\right\|_{H}+\left\|R_{n}(F(X(s)))-F(X(s))\right\|_{H} \\
(19) & \leq\left\|R_{n}\right\|_{H}\left\|F\left(X_{n}(s)\right)-F(X(s))\right\|_{H}+\left\|\left(R_{n}-I\right) F(X(s))\right\|_{H} .
\end{aligned}
$$

Therefore $R_{n}\left(F\left(X_{n}(s)\right)\right) \rightarrow F(X(s))$ because of the uniform boundedness of $\left\|R_{n}\right\|_{\mathcal{L}(H)}$, and the convergence of $\left(R_{n}-I\right) x \rightarrow 0$. So, by (17) and Lebesgue dominated convergence theorem,

$$
\int_{0}^{t}\left\langle\Psi_{x}\left(s, X_{n}^{\xi}(s)\right), R_{n} F\left(X_{n}^{\xi}(s)\right)\right\rangle_{H} d s \rightarrow \int_{0}^{t}\left\langle\Psi_{x}\left(s, X^{\xi}(s)\right), F\left(X^{\xi}(s)\right)\right\rangle_{H} d s
$$

We will discuss the convergence of the term,

$$
\int_{0}^{t}\left\langle\Psi_{x}\left(s, X_{n}^{\xi}(s)\right), A X_{n}^{\xi}(s)\right\rangle_{H} d s
$$

at the end.

Now consider the third term of the R.H.S. of eq. (16),

$$
\int_{0}^{t} \frac{1}{2} \operatorname{tr}\left(\Psi_{x x}\left(s, X_{n}^{\xi}(s)\right)\left(R_{n} B\left(X_{n}^{\xi}(s)\right)\right) Q\left(R_{n} B\left(X_{n}^{\xi}(s)\right)\right)^{*}\right) d s .
$$

We have

$$
\begin{aligned}
& \operatorname{tr}\left(\Psi_{x x}\left(s, X_{n}^{\xi}(s)\right)\left(R_{n} B\left(X_{n}^{\xi}(s)\right)\right) Q\left(R_{n} B\left(X_{n}^{\xi}(s)\right)\right)^{*}\right) \\
& =\operatorname{tr}\left(\left(R_{n} B\left(X_{n}^{\xi}(s)\right)\right)^{*} \Psi_{x x}\left(s, X_{n}^{\xi}(s)\right)\left(R_{n} B\left(X_{n}^{\xi}(s)\right)\right) Q\right) \\
& =\sum_{j=1}^{\infty} \lambda_{j}\left\langle\Psi_{x x}\left(s, X_{n}^{\xi}(s)\right)\left(R_{n} B\left(X_{n}^{\xi}(s)\right)\right) f_{j},\left(R_{n} B\left(X_{n}^{\xi}(s)\right)\right) f_{j}\right\rangle_{H} .
\end{aligned}
$$

Here we used the property that, for a symmetric operator $T \in \mathcal{L}(H)$ and $\phi \in \mathcal{L}(K, H)$,

$$
\operatorname{tr}\left(T \phi Q \phi^{*}\right)=\operatorname{tr}\left(\phi^{*} T \phi Q\right) .
$$

$\Psi_{x x}$ being continuous, $B$ is continuous, $\left\|R_{n}\right\|_{\mathcal{L}(H)}$ is uniformly bounded and having the convergence of $\left(R_{n}-I\right) x \rightarrow 0$, by a similar calculation as in (19) we deduce that 


$$
\begin{aligned}
& \left\langle\Psi_{x x}\left(s, X_{n}^{\xi}(s)\right)\left(R_{n} B\left(X_{n}^{\xi}(s)\right)\right) f_{j},\left(R_{n} B\left(X_{n}^{\xi}(s)\right)\right) f_{j}\right\rangle_{H} \\
& \rightarrow\left\langle\Psi_{x x}\left(s, X^{\xi}(s)\right)\left(B\left(X^{\xi}(s)\right)\right) f_{j},\left(B\left(X^{\xi}(s)\right)\right) f_{j}\right\rangle_{H} .
\end{aligned}
$$

Hence,

$$
\begin{aligned}
& \operatorname{tr}\left(\Psi_{x x}\left(s, X_{n}^{\xi}(s)\right)\left(R_{n} B\left(X_{n}^{\xi}(s)\right)\right) Q\left(R_{n} B\left(X_{n}^{\xi}(s)\right)\right)^{*}\right) \\
& \rightarrow \operatorname{tr}\left(\Psi_{x x}\left(s, X^{\xi}(s)\right)\left(B\left(X^{\xi}(s)\right)\right) Q\left(B\left(X^{\xi}(s)\right)\right)^{*}\right) .
\end{aligned}
$$

Also we have,

$$
\begin{aligned}
\operatorname{tr}\left(\Psi_{x x}\left(s, X_{n}^{\xi}(s)\right)\left(R_{n} B\left(X_{n}^{\xi}(s)\right)\right) Q\left(R_{n} B\left(X_{n}^{\xi}(s)\right)\right)^{*}\right) & \leq\left\|\Psi_{x x}\left(s, X_{n}^{\xi}(s)\right)\right\|\left\|R_{n} B\left(X_{n}^{\xi}(s)\right)\right\|^{2} \\
\text { by }(\mathrm{A} 3) & \leq\left\|\Psi_{x x}\left(s, X_{n}^{\xi}(s)\right)\right\|\left\|R_{n}\right\|^{2} l\left(1+\left\|X_{n}^{\xi}(s)\right\|^{2}\right) .
\end{aligned}
$$

So by (17) and Lebesgue dominated convergence theorem we conclude that,

$$
\begin{aligned}
& \int_{0}^{t} \operatorname{tr}\left(\Psi_{x x}\left(s, X_{n}^{\xi}(s)\right)\left(R_{n} B\left(X_{n}^{\xi}(s)\right)\right) Q\left(R_{n} B\left(X_{n}^{\xi}(s)\right)\right)^{*}\right) d s \\
& \rightarrow \int_{0}^{t} \operatorname{tr}\left(\Psi_{x x}\left(s, X^{\xi}(s)\right)\left(B\left(X^{\xi}(s)\right)\right) Q\left(B\left(X^{\xi}(s)\right)\right)^{*}\right) d s,
\end{aligned}
$$

$P$-a.s.

Now consider the fourth term of the R.H.S. of eq. (16),

$\int_{H \backslash\{0\}}\left[\Psi\left(s, X_{n}^{\xi}(s)+R_{n} f\left(v, X_{n}^{\xi}(s)\right)\right)-\Psi\left(s, X_{n}^{\xi}(s)\right)-\left\langle\Psi_{x}\left(s, X_{n}^{\xi}(s)\right), R_{n} f\left(v, X_{n}^{\xi}(s)\right)\right\rangle_{H}\right] \beta(d v)$.

Using Theorem [3. (17), the continuity of $\Psi, \Psi_{x}, f$ and $\left(R_{n}-I\right) x \rightarrow 0$, we conclude

$$
\left[\Psi\left(s, X_{n}^{\xi}(s)+R_{n} f\left(v, X_{n}^{\xi}(s)\right)\right)-\Psi\left(s, X_{n}^{\xi}(s)\right)-\left\langle\Psi_{x}\left(s, X_{n}^{\xi}(s)\right), R_{n} f\left(v, X_{n}^{\xi}(s)\right)\right\rangle_{H}\right]
$$

converges to

$$
\left[\Psi\left(s, X^{\xi}(s)+f\left(v, X^{\xi}(s)\right)\right)-\Psi\left(s, X^{\xi}(s)\right)-\left\langle\Psi_{x}\left(s, X^{\xi}(s)\right), f\left(v, X^{\xi}(s)\right)\right\rangle_{H}\right]
$$

$P$-a.s.. Again by Taylor's theorem, the Cauchy Schwarz inequality and assumption (8), we get 


$$
\begin{aligned}
& \int_{H \backslash\{0\}}\left|\Psi\left(s, X_{n}^{\xi}(s)+R_{n} f\left(v, X_{n}^{\xi}(s)\right)\right)-\Psi\left(s, X_{n}^{\xi}(s)\right)-\left\langle\Psi_{x}\left(s, X_{n}^{\xi}(s)\right), R_{n} f\left(v, X_{n}^{\xi}(s)\right)\right\rangle_{H}\right| \beta(d v) \\
& =\int_{H \backslash\{0\}}\left|\int_{0}^{1} \Psi_{x x}\left(s, X_{n}^{\xi}(s)+\theta R_{n} f\left(v, X_{n}^{\xi}(s)\right)\right)\left\langle R_{n} f\left(v, X_{n}^{\xi}(s)\right), R_{n} f\left(v, X_{n}^{\xi}(s)\right)\right\rangle d \theta\right| \beta(d v) \\
& \leq \int_{H \backslash\{0\}} \int_{0}^{1}\left\|\Psi_{x x}\left(s, X_{n}^{\xi}(s)+\theta R_{n} f\left(v, X_{n}^{\xi}(s)\right)\right)\right\|\left\|R_{n} f\left(v, X_{n}^{\xi}(s)\right)\right\|^{2} d \theta \beta(d v) \\
& \leq \int_{H \backslash\{0\}} \int_{0}^{1} h_{2}\left(\left\|X_{n}^{\xi}(s)+\theta R_{n} f\left(v, X_{n}^{\xi}(s)\right)\right\|\right)\left\|R_{n} f\left(v, X_{n}^{\xi}(s)\right)\right\|^{2} d \theta \beta(d v) \\
& \leq C \int_{H \backslash\{0\}} \int_{0}^{1}\left(h_{2}\left(\left\|X_{n}^{\xi}(s)\right\|\right)+C h_{2}(\theta) h_{2}\left(\left\|R_{n} f\left(v, X_{n}^{\xi}(s)\right)\right\|\right)\right)\left\|R_{n} f\left(v, X_{n}^{\xi}(s)\right)\right\|^{2} d \theta \beta(d v) \\
& \leq C \int_{H \backslash\{0\}} h_{2}\left(\left\|X_{n}^{\xi}(s)\right\|\right)\left\|R_{n} f\left(v, X_{n}^{\xi}(s)\right)\right\|^{2} \beta(d v) \\
& \quad+C^{2} h_{2}(1) \int_{H \backslash\{0\}} h_{2}\left(\left\|R_{n} f\left(v, X_{n}^{\xi}(s)\right)\right\|\right)\left\|R_{n} f\left(v, X_{n}^{\xi}(s)\right)\right\|^{2} \beta(d v)<\infty,
\end{aligned}
$$

$P$-a.s. by condition (9). Since $\left\|R_{n}\right\|_{\mathcal{L}(H)}$ is uniformly bounded, therefore by Lebesgue dominated convergence theorem

$\int_{0}^{t} \int_{H \backslash\{0\}}\left[\Psi\left(s, X_{n}^{\xi}(s)+R_{n} f\left(v, X_{n}^{\xi}(s)\right)\right)-\Psi\left(s, X_{n}^{\xi}(s)\right)-\left\langle\Psi_{x}\left(s, X_{n}^{\xi}(s)\right), R_{n} f\left(v, X_{n}^{\xi}(s)\right)\right\rangle_{H}\right] \beta(d v) d s$ converges to

$$
\begin{aligned}
\int_{0}^{t} \int_{H \backslash\{0\}}\left[\Psi\left(s, X^{\xi}(s)+f\left(v, X^{\xi}(s)\right)\right)-\Psi\left(s, X^{\xi}(s)\right)-\left\langle\Psi_{x}\left(s, X^{\xi}(s)\right), f\left(v, X^{\xi}(s)\right)\right\rangle_{H}\right] \beta(d v) d s \\
\quad P \text {-a.s.. }
\end{aligned}
$$

Now consider the fifth term of the R.H.S. of eq. (16),

$$
\begin{gathered}
\int_{0}^{t}\left\langle\Psi_{x}\left(s, X_{n}^{\xi}(s)\right), R_{n} B\left(X_{n}^{\xi}(s)\right) d W_{s}\right\rangle_{H} \cdot \\
E\left|\int_{0}^{t}\left\langle\Psi_{x}\left(s, X_{n}^{\xi}(s)\right), R_{n} B\left(X_{n}^{\xi}(s)\right) d W_{s}\right\rangle_{H}-\int_{0}^{t}\left\langle\Psi_{x}\left(s, X^{\xi}(s)\right), B\left(X^{\xi}(s)\right) d W_{s}\right\rangle_{H}\right|^{2} \\
\leq C \int_{0}^{t} E \|\left(B\left(X^{\xi}(s)\right)\right)^{*}\left(\Psi_{x}\left(s, X_{n}^{\xi}(s)-\Psi_{x}\left(s, X^{\xi}(s)\right)\right) \|_{\mathcal{L}_{2}\left(K_{Q}, H\right)}^{2} d s\right. \\
+C \int_{0}^{t} E\left\|\left(\left(B\left(X^{\xi}(s)\right)\right)^{*}-\left(R_{n} B\left(X_{n}^{\xi}(s)\right)\right)^{*}\right) \Psi_{x}\left(s, X_{n}^{\xi}(s)\right)\right\|_{\mathcal{L}_{2}\left(K_{Q}, H\right)}^{2} d s \\
\leq C \int_{0}^{t} E\left(\|\left(B\left(X^{\xi}(s)\right)\left\|_{\mathcal{L}_{2}\left(K_{Q}, H\right)}^{2}\right\| \Psi_{x}\left(s, X_{n}^{\xi}(s)\right)-\Psi_{x}\left(s, X^{\xi}(s)\right) \|_{H}^{2}\right) d s\right. \\
+C \int_{0}^{t} E\left(\left\|\left(B\left(X^{\xi}(s)\right)\right)^{*}-\left(R_{n} B\left(X_{n}^{\xi}(s)\right)\right)^{*}\right\|_{\mathcal{L}_{2}\left(K_{Q}, H\right)}^{2}\left\|\Psi_{x}\left(s, X_{n}^{\xi}(s)\right)\right\|_{H}^{2}\right) d s .
\end{gathered}
$$


Here, the first integral converges to zero, since the first factor is an integrable process, and the second factor converges to zero almost surely, so we can apply Lebesgue dominated convergence theorem. The second integral is bounded by $M\left\|\left(B\left(X^{\xi}(s)\right)\right)^{*}-\left(R_{n} B\left(X_{n}^{\xi}(s)\right)\right)^{*}\right\|_{\Lambda_{2}\left(K_{Q}, H\right)}^{2}$ for some constant $M$ (from (17), $\Psi_{x}$ is bounded by some constant), since $R_{n} B\left(X_{n}^{\xi}(s)\right) \rightarrow B\left(X^{\xi}(s)\right)$ in the space $\Lambda_{2}\left(K_{Q}, H\right)$, so the second integral also converges to zero by Lebesgue dominated convergence theorem. Hence we conclude that,

$$
\int_{0}^{t}\left\langle\Psi_{x}\left(s, X_{n}^{\xi}(s)\right), R_{n} B\left(X_{n}^{\xi}(s)\right) d W_{s}\right\rangle_{H} \rightarrow \int_{0}^{t}\left\langle\Psi_{x}\left(s, X^{\xi}(s)\right), B\left(X^{\xi}(s)\right) d W_{s}\right\rangle_{H}
$$

in mean square, therefore in probability.

Now consider the sixth term of the R.H.S. of eq. (16),

$$
\begin{gathered}
\int_{0}^{t} \int_{H \backslash\{0\}}\left[\Psi\left(s, X_{n}^{\xi}(s)+R_{n} f\left(v, X_{n}^{\xi}(s)\right)\right)-\Psi\left(s, X_{n}^{\xi}(s)\right)\right] q(d v, d s) . \\
\left|\left\{\Psi\left(s, X_{n}^{\xi}(s)+R_{n} f\left(v, X_{n}^{\xi}(s)\right)\right)-\Psi\left(s, X_{n}^{\xi}(s)\right)\right\}-\left\{\Psi\left(s, X^{\xi}(s)+f\left(v, X^{\xi}(s)\right)\right)-\Psi\left(s, X^{\xi}(s)\right)\right\}\right|^{2} \\
=\left|\left[\Psi\left(s, X_{n}^{\xi}(s)+R_{n} f\left(v, X_{n}^{\xi}(s)\right)\right)-\Psi\left(s, X^{\xi}(s)+f\left(v, X^{\xi}(s)\right)\right)\right]+\left[\Psi\left(s, X^{\xi}(s)\right)-\Psi\left(s, X_{n}^{\xi}(s)\right)\right]\right|^{2} \\
\leq 2\left|\Psi\left(s, X_{n}^{\xi}(s)+R_{n} f\left(v, X_{n}^{\xi}(s)\right)\right)-\Psi\left(s, X^{\xi}(s)+f\left(v, X^{\xi}(s)\right)\right)\right|^{2} \\
\quad+2\left|\Psi\left(s, X^{\xi}(s)\right)-\Psi\left(s, X_{n}^{\xi}(s)\right)\right|^{2} \\
\leq 2\left\|X_{n}^{\xi}(s)+R_{n} f\left(v, X_{n}^{\xi}(s)\right)-\left\{X^{\xi}(s)+f\left(v, X^{\xi}(s)\right)\right\}\right\|^{2} \sup _{0<\theta \leq 1}\left\|\Psi_{x}\left(s, \eta_{1}(\theta)\right)\right\|^{2} \\
\quad+2\left\|X^{\xi}(s)-X_{n}^{\xi}(s)\right\|^{2} \sup _{0<\theta \leq 1}\left\|\Psi_{x}\left(s, \eta_{2}(\theta)\right)\right\|^{2}
\end{gathered}
$$

to obtain the above inequality, we used the following inequality

$$
\|\Psi(x)-\Psi(y)\| \leq\|x-y\| \sup _{0<\theta \leq 1}\left\|\Psi_{x}(y+\theta(x-y))\right\| .
$$

Where

$$
\eta_{1}(\theta)=X^{\xi}(s)+f\left(v, X^{\xi}(s)\right)+\theta\left(X_{n}^{\xi}(s)-X^{\xi}(s)+R_{n} f\left(v, X_{n}^{\xi}(s)\right)-f\left(v, X^{\xi}(s)\right)\right)
$$

and

$$
\eta_{2}(\theta)=X_{n}^{\xi}(s)+\theta\left(X^{\xi}(s)-X_{n}^{\xi}(s)\right) .
$$

Therefore, by using condition (77), we can write the above inequality is

$$
\begin{aligned}
& \left|\left\{\Psi\left(s, X_{n}^{\xi}(s)+R_{n} f\left(v, X_{n}^{\xi}(s)\right)\right)-\Psi\left(s, X_{n}^{\xi}(s)\right)\right\}-\left\{\Psi\left(s, X^{\xi}(s)+f\left(v, X^{\xi}(s)\right)\right)-\Psi\left(s, X^{\xi}(s)\right)\right\}\right|^{2} \\
\leq & 4\left\{\left\|X_{n}^{\xi}(s)-X^{\xi}(s)\right\|^{2}+\left\|R_{n} f\left(v, X_{n}^{\xi}(s)\right)-f\left(v, X^{\xi}(s)\right)\right\|^{2}\right\} \sup _{0<\theta \leq 1}\left\{h_{1}\left(\left\|\eta_{1}(\theta)\right\|\right)\right\}^{2} \\
& +2\left\|X^{\xi}(s)-X_{n}^{\xi}(s)\right\|^{2} \sup _{0<\theta \leq 1}\left\{h_{1}\left(\left\|\eta_{2}(\theta)\right\|\right)\right\}^{2} .
\end{aligned}
$$

Now as $n \rightarrow \infty$, the R.H.S. of the above inequality converges to $0 P$-a.s.. Therefore 


$$
\begin{aligned}
& \lim _{n \rightarrow \infty} \int_{0}^{t} \int_{H \backslash\{0\}} \|\left\{\Psi\left(s, X_{n}^{\xi}(s)+R_{n} f\left(v, X_{n}^{\xi}(s)\right)\right)-\Psi\left(s, X_{n}^{\xi}(s)\right)\right\} \\
& -\left\{\Psi\left(s, X^{\xi}(s)+f\left(v, X^{\xi}(s)\right)\right)-\Psi\left(s, X^{\xi}(s)\right)\right\} \|^{2} \beta(d v) d s=0
\end{aligned}
$$

$P$-a.s.. Again by Taylor's theorem, the Cauchy -Schwarz inequality and assumption (7) we get

$$
\begin{aligned}
& \int_{0}^{t} \int_{H \backslash\{0\}}\left|\Psi\left(s, X_{n}^{\xi}(s)+R_{n} f\left(v, X_{n}^{\xi}(s)\right)\right)-\Psi\left(s, X_{n}^{\xi}(s)\right)\right|^{2} \beta(d v) d s \\
& =\int_{0}^{t} \int_{H \backslash\{0\}}\left|\int_{0}^{1} \Psi_{x}\left(s, X_{n}^{\xi}(s)+\theta R_{n} f\left(v, X_{n}^{\xi}(s)\right)\right) R_{n} f\left(v, X_{n}^{\xi}(s)\right) d \theta\right|^{2} \beta(d v) d s \\
& \leq \int_{0}^{t} \int_{H \backslash\{0\}} \int_{0}^{1}\left\|\Psi_{x}\left(s, X_{n}^{\xi}(s)+\theta R_{n} f\left(v, X_{n}^{\xi}(s)\right)\right)\right\|^{2}\left\|R_{n} f\left(v, X_{n}^{\xi}(s)\right)\right\|^{2} d \theta \beta(d v) d s \\
& \leq \int_{0}^{t} \int_{H \backslash\{0\}} \int_{0}^{1} h_{1}\left(\left\|X_{n}^{\xi}(s)+\theta R_{n} f\left(v, X_{n}^{\xi}(s)\right)\right\|\right)^{2}\left\|R_{n} f\left(v, X_{n}^{\xi}(s)\right)\right\|^{2} d \theta \beta(d v) d s \\
& \leq C^{2} \int_{0}^{t} \int_{H \backslash\{0\}} \int_{0}^{1}\left\{h_{1}\left(\left\|X_{n}^{\xi}(s)\right\|\right)+C h_{1}(\theta) h_{1}\left(\left\|R_{n} f\left(v, X_{n}^{\xi}(s)\right)\right\|\right)\right\}^{2}\left\|R_{n} f\left(v, X_{n}^{\xi}(s)\right)\right\|^{2} d \theta \beta(d v) d s \\
& \leq 2 C^{2} \int_{0}^{t} \int_{H \backslash\{0\}} h_{1}\left(\left\|X_{n}^{\xi}(s)\right\|\right)^{2}\left\|R_{n} f\left(v, X_{n}^{\xi}(s)\right)\right\|^{2} \beta(d v) d s
\end{aligned}
$$

$$
+2 C^{4} h_{1}(1) \int_{0}^{t} \int_{H \backslash\{0\}} h_{1}\left(\left\|R_{n} f\left(v, X_{n}^{\xi}(s)\right)\right\|\right)^{2}\left\|R_{n} f\left(v, X_{n}^{\xi}(s)\right)\right\|^{2} \beta(d v) d s<\infty,
$$

$P$-a.s. by the condition (91). Therefore from (20) and (21) we conclude that

$$
\begin{aligned}
& \lim _{n \rightarrow \infty} \int_{0}^{t} \int_{H \backslash\{0\}}\left[\Psi\left(s, X_{n}^{\xi}(s)+R_{n} f\left(v, X_{n}^{\xi}(s)\right)\right)-\Psi\left(s, X_{n}^{\xi}(s)\right)\right] q(d v, d s) \\
& =\int_{0}^{t} \int_{H \backslash\{0\}}\left[\Psi\left(s, X^{\xi}(s)+f\left(v, X^{\xi}(s)\right)\right)-\Psi\left(s, X^{\xi}(s)\right)\right] q(d v, d s)
\end{aligned}
$$

in probability.

Thus we have showed the term by term convergence of left- and right- hand sides of eq. (16) except for the term $\int_{0}^{t}\left\langle\Psi_{x}\left(s, X_{n}^{\xi}(s)\right), A X_{n}^{\xi}(s)\right\rangle_{H} d s$. Now since all the terms of the eq. (16) converge, so the term $\int_{0}^{t}\left\langle\Psi_{x}\left(s, X_{n}^{\xi}(s)\right), A X_{n}^{\xi}(s)\right\rangle_{H} d s$ has to converge. Where the nonstochastic integrals converge in $P$-a.s. sense and stochastic integrals converge in probability. In conclusion, possibly for a subsequence of leftand right- hand sides of eq. (16) converges in $P$-a.s. sense for all $t \in[0, T]$. Hence we conclude that eq. (13) converges $P$-a.s. and we can write our Itô formula for mild solutions as $\lim _{n \rightarrow \infty} \int_{0}^{t}\left\langle\Psi_{x}\left(s, X_{n}^{\xi}(s)\right), A X_{n}^{\xi}(s)\right\rangle_{H} d s$ i.e. (15). This completes the proof. 
Remark 4. When the mild solution satisfies $X^{\xi}(t) \in \mathcal{D}(A)$, then $A X^{\xi}(t)$ is well defined. Hence, we get back the same Itô formula for strong solutions as in eq. (13), as by Theorem 0 when $X^{\xi}(t) \in \mathcal{D}(A)$, then $X^{\xi}(t)$ is also a strong solution.

In the next section we will use the following

Corollary 2. Assume that all assumptions in Theorem 4 are satisfied. Let $X_{n}^{\xi}$ be the Yosida approximation of the mild solution $X^{\xi}$ of (11), i.e let $X_{n}^{\xi}$ be the strong solution of (3), then

$$
\lim _{n \rightarrow \infty}\left|\mathcal{L} \Psi\left(s, X_{n}^{\xi}(s)\right)-\mathcal{L}_{n} \Psi\left(s, X_{n}^{\xi}(s)\right)\right|=0 \quad P-\text { a.s. }
$$

Proof. As we assume $\xi \in \mathcal{D}(A)$ a.s., and make the assumptions that (7), (8), (9) hold, the operators $\mathcal{L}$ in (11), and $\mathcal{L}_{n}$ in (14) are well defined in all their terms.

$$
\begin{aligned}
& \lim _{n \rightarrow \infty}\left|\mathcal{L} \Psi\left(s, X_{n}^{\xi}(s)\right)-\mathcal{L}_{n} \Psi\left(s, X_{n}^{\xi}(s)\right)\right| \\
= & \lim _{n \rightarrow \infty} \mid\left\langle\Psi_{x}\left(s, X_{n}^{\xi}(s)\right), A X_{n}^{\xi}(s)\right\rangle \\
+ & \left\langle\Psi_{x}\left(s, X_{n}^{\xi}(s)\right), F\left(X^{\xi}(s)\right)\right\rangle_{H}+\frac{1}{2} \operatorname{tr}\left(\Psi_{x x}\left(s, X_{n}^{s, \xi}(s)\right)\left(B\left(X_{n}^{\xi}(s)\right)\right) Q\left(B\left(X_{n}^{\xi}(s)\right)\right)^{*}\right) \\
& +\int_{H \backslash\{0\}}\left[\Psi\left(s, X_{n}^{\xi}(s)+f\left(v, X_{n}^{\xi}(s)\right)\right)-\Psi\left(s, X_{n}^{\xi}(s)\right)-\left\langle\Psi_{x}\left(s, X_{n}^{\xi}(s)\right), f\left(v, X_{n}^{\xi}(s)\right)\right\rangle_{H}\right] \beta(d v) \\
- & \left\langle\Psi_{x}\left(s, X_{n}^{\xi}(s)\right), A X_{n}^{\xi}(s)\right\rangle-\left\langle\Psi_{x}\left(s, X_{n}^{\xi}(s)\right), R_{n} F\left(X_{n}^{\xi}(s)\right)\right\rangle_{H} \\
- & \frac{1}{2} \operatorname{tr}\left(\Psi_{x x}\left(s, X_{n}^{\xi}(s)\right)\left(R_{n} B\left(X_{n}^{\xi}(s)\right)\right) Q\left(R_{n} B\left(X_{n}^{\xi}(s)\right)\right)^{*}\right) \\
- & \int_{H \backslash\{0\}}\left[\Psi\left(s, X_{n}^{\xi}(s)+R_{n} f\left(v, X_{n}^{\xi}(s)\right)\right)-\Psi\left(s, X_{n}^{\xi}(s)\right)-\left\langle\Psi_{x}\left(s, X_{n}^{\xi}(s)\right), R_{n} f\left(v, X_{n}^{\xi}(s)\right)\right\rangle_{H}\right] \beta(d v) \mid . \\
= & \lim _{n \rightarrow \infty}\left\langle\Psi_{x}\left(s, X_{n}^{\xi}(s)\right), A X_{n}^{\xi}(s)\right\rangle-\lim _{n \rightarrow \infty}\left\langle\Psi_{x}\left(s, X_{n}^{\xi}(s)\right), A X_{n}^{\xi}(s)\right\rangle \mid=0 \quad P-a . s . .
\end{aligned}
$$

Similarly as in Theorem 4the r.h.s. of (23) can be divided in terms which modulus converges to zero a.s.. This is proven using Theorem 3 , the fact that $\left\|R_{n}\right\|$ is uniformly bounded, as well as $\left(R_{n}-I\right) x \rightarrow 0$, that (17) holds, $E\left[\sup _{0 \leq s \leq T}\left\|X^{\xi}(s)\right\|_{H}^{2}\right]<$ $\infty$ and conditions (7), (8), (9) hold.

Here we will present an example of Theorem 4 .

\section{Example 1:-}

Let $\Lambda: H \rightarrow \mathbb{R}$. Assume that the assumptions of Theorem 4 hold. Now if we apply the Itô formula for mild solutions, of (1), for the function $e^{c t} \Lambda(x)$ where $c>0$, 
$t \geq 0$ then it will be

$$
\begin{aligned}
& \lim _{n \rightarrow \infty} \int_{0}^{t} e^{c s}\left\langle\Lambda^{\prime}\left(X_{n}^{\xi}(s)\right), A X_{n}^{\xi}(s)\right\rangle_{H} d s \\
& =e^{c t} \Lambda\left(X^{\xi}(t)\right)-\Lambda(\xi)-\int_{0}^{t} c e^{c s}\left(\Lambda\left(X^{\xi}(s)\right)\right) d s \\
& -\int_{0}^{t} e^{c s}\left\langle\Lambda^{\prime}\left(X^{\xi}(s)\right), F\left(X^{\xi}(s)\right)\right\rangle_{H} d s-\int_{0}^{t} e^{c s} \frac{1}{2} \operatorname{tr}\left(\Lambda^{\prime \prime}\left(X^{\xi}(s)\right)\left(B\left(X^{\xi}(s)\right)\right) Q\left(B\left(X^{\xi}(s)\right)\right)^{*}\right) d s \\
& -\int_{0}^{t} \int_{H \backslash\{0\}} e^{c s}\left[\Lambda\left(X^{\xi}(s)+f\left(v, X^{\xi}(s)\right)\right)-\Lambda\left(X^{\xi}(s)\right)-\left\langle\Lambda^{\prime}\left(X^{\xi}(s)\right), f\left(v, X^{\xi}(s)\right)\right\rangle_{H}\right] \beta(d v) d s \\
& -\int_{0}^{t} e^{c s}\left\langle\Lambda^{\prime}\left(X^{\xi}(s)\right), B\left(X^{\xi}(s)\right) d W_{s}\right\rangle_{H} \\
& -\int_{0}^{t} \int_{H \backslash\{0\}} e^{c s}\left[\Lambda\left(X^{\xi}(s)+f\left(v, X^{\xi}(s)\right)\right)-\Lambda\left(X^{\xi}(s)\right)\right] q(d v, d s) \\
& P \text {-a.s.. }
\end{aligned}
$$

Remark 5. By Corollary 1 we can take in Example 1 e.g. $\Lambda \in C_{b}^{2}(\mathbb{R} \times H)$, but it can also be a function in $C^{2}(\mathbb{R} \times H)$ for which (7), (8) and (9) are satisfied. Suppose e.g. $\int_{H \backslash\{0\}}\left(\|f(v, x)\|^{2}+\|f(v, x)\|^{4}\right) \beta(d v)<\infty$, for all $x \in H$ and then choose e.g. $\Lambda(x)=\|x\|^{2}$ (see the examples in [19], page 184).

3.4. Applications of the Itô formula obtained by Yosida approximations: Stability properties of mild solutions. In this section we will present how the Itô formula obtained by Yosida approximation provides a good method to prove that the mild solution of (1) is "exponential stable in the mean square sense" ("exponential stable in the m.s.s") or "exponential ultimate bounded in the mean square sense" ("exponential ultimate bounded in the m.s.s.") under suitable conditions. In the whole section we assume that conditions (A1), (A2) and (A3) hold. Then due to Theorem 1 there exists a unique mild solution $\left\{X^{x}(t), t \geq 0\right\}$ of (1D) with initial condition $X^{x}(0)=x \in H$.

Definition 5. We say that that the solution of (11) is exponentially stable in the mean square sense (m.s.s.) if there exist $c, \beta>0$ such that for all $t \geq 0$ and $x \in H$,

$$
E\left\|X^{x}(t)\right\|_{H}^{2} \leq c e^{-\beta t}\|x\|_{H}^{2} ; .
$$

Here as usual $C^{2}(H)$ denotes the space of continuous functions $\Psi: H \rightarrow \mathbb{R}$, with continuous first and second Fréchet derivative $\Psi^{\prime}(x)$ and $\Psi^{\prime \prime}(x), x \in H$.

Definition 6. Let $\mathcal{L}$ be defined as in (11). A function $\Psi: H \rightarrow \mathbb{R} \in C^{2}(H)$ is called a Lyapunov function for $\mathcal{L}$ if it satisfies the following conditions:

(I) There exist finite constants $c_{1}, c_{2}>0$ such that; for all $x \in H$

$$
c_{1}\|x\|_{H}^{2} \leq \Psi(x) \leq c_{2}\|x\|_{H}^{2}
$$

(II) There exists a constants $c_{3}>0$ such that

$$
\mathcal{L} \Psi(x) \leq-c_{3} \Psi(x) \quad \text { for all } \quad x \in \mathcal{D}(A)
$$


Theorem 5. Assume there exists a function $\Psi \in C^{2}(H)$ which is a Lyapunov function for $\mathcal{L}$, and such that (77), (8) and (9) are satisfied, then the mild solution of (11) is exponentially stable in the m.s.s.

If we assume that the first two Frechét derivatives of the Lyapunov function $\Psi$ are uniformly bounded, i.e. $\Psi \in C_{b}^{2}(H)$, then due to Remark 2 it follows directly that the following Corollary holds:

Corollary 3. If there exists a function $\Psi \in C_{b}^{2}(H)$ which is a Lyapunov function for $\mathcal{L}$, then the mild solution of (10) is exponentially stable in the m.s.s.

A detailed proof of Corollary [3] is in Theorem 6.4 of [13], Theorem 4.2 of [20] (see also Section 7.1 in [24]). Here we present a simplified proof of the more general statement in Theorem 5 by using the limiting argument of Theorem 4 and Corollary [2.

Proof. We first prove that inequality (24) holds for all $x \in \mathcal{D}(A)$.

By applying the Itô formula to the Yosida approximation $X_{n}^{x}$ of $X^{x}$ and by taking expectations on both sides we obtain

$e^{c_{3} t} E \Psi\left(X_{n}^{x}(t)\right)-\Psi\left(X_{n}^{x}(0)\right)=E \int_{0}^{t} e^{c_{3} s}\left(c_{3} \Psi\left(X_{n}^{x}(s)\right)+\mathcal{L}_{n} \Psi\left(X_{n}^{x}(s)\right)\right) d s . \quad \forall x \in \mathcal{D}(A)$.

From condition (II),

$$
c_{3} \Psi\left(X_{n}^{x}(s)\right)+\mathcal{L}_{n} \Psi\left(X_{n}^{x}(s)\right) \leq-\mathcal{L} \Psi\left(X_{n}^{x}(s)\right)+\mathcal{L}_{n} \Psi\left(X_{n}^{x}(s)\right) \quad \forall x \in \mathcal{D}(A) .
$$

$$
\Rightarrow e^{c_{3} t} E \Psi\left(X_{n}^{x}(t)\right)-\Psi\left(X_{n}^{x}(0)\right) \leq E \int_{0}^{t} e^{c_{3} s}\left(-\mathcal{L} \Psi\left(X_{n}^{x}(s)\right)+\mathcal{L}_{n} \Psi\left(X_{n}^{x}(s)\right)\right) d s . \quad \forall x \in \mathcal{D}(A) .
$$

Using Corollary 2 and (17) we obtain

$$
e^{c_{3} t} E \Psi\left(X^{x}(t)\right) \leq \Psi(x)
$$

$\Rightarrow c_{1} E\left\|X^{x}(t)\right\|_{H}^{2} \leq E \Psi\left(X^{x}(t)\right) \leq e^{-c_{3} t} \Psi(x) \leq c_{2} e^{-c_{3} t}\|x\|_{H}^{2} \quad$ [from condition (ii)]

$$
\Rightarrow E\left\|X^{x}(t)\right\|_{H}^{2} \leq \frac{c_{2}}{c_{1}} e^{-c_{3} t}\|x\|_{H}^{2} \forall x \in \mathcal{D}(A) .
$$

Choosing $c=\frac{c_{2}}{c_{1}}$ and $\beta=c_{3}$, we can conclude that (24) holds for all $x \in \mathcal{D}(A)$. As however $\mathcal{D}(A)$ is dense in $H$ and, according to Corollary 5.3.2 in [24, for each $t>0$ there exists a constant $C_{t}$ such that

$$
\mathbb{E}\left\|X^{x}(t)-X^{y}(t)\right\|_{H}^{2} \leq C_{t}\|x-y\|_{H}^{2},
$$

we can conclude that, the mild solution $X^{x}(t)$ is exponentially stable in the mean square sense (m.s.s.) 
Definition 7. We say that the mild solution of (10) is exponentially ultimate bounded in the mean square sense (m.s.s.) if there exist positive constants $c, \beta, M$ such that

$$
E\left\|X^{x}(t)\right\|_{H}^{2} \leq c e^{-\beta t}\|x\|_{H}^{2}+M ; \quad \text { for all } x \in H \text { andt }>0 .
$$

Theorem 6. Let us assume there exists a function $\Psi \in C^{2}(H)$ satisfying the following conditions:

(i) $c_{1}\|x\|_{H}^{2}-k_{1} \leq \Psi(x) \leq c_{2}\|x\|_{H}^{2}-k_{2}$; for all $x \in H$

(ii) $\mathcal{L} \Psi \leq-c_{3} \Psi(x)+k_{3}$; for $x \in \mathcal{D}(A)$,

where $c_{1}, c_{2}, c_{3}, k_{1}, k_{2}$ and $k_{3}$ are finite, positive constants.

Moreover, let us assume (7), (8) and (9) are satisfied.

Then the mild solution $\left\{X^{x}(t), t \geq 0\right\}$ of (11) is exponentially ultimate bounded in the m.s.s.

By similar arguments as in the proof of Corollary 3 the following Corollary follows directly

Corollary 4. If there exists a function $\Psi \in C_{b}^{2}(H)$ such that conditions ( $i$ ) and (ii) hold in Theorem [6, then the mild solution $\left\{X^{x}(t), t \geq 0\right\}$ of (11) is exponentially ultimate bounded in the m.s.s..

A detailed proof of Corollary 4 is in Theorem 7.1 of 13 , Theorem 5.2 of 20 (see also Section 7.2 in [24]). Here we present a simplified proof of the more general statement in Theorem 6 by using the limiting argument of Theorem 4 and Corollary 2

Proof. First let us assume $x \in \mathcal{D}(A)$. By applying the Itô formula to the Yosida approximation $X_{n}^{x}$ of $X^{x}$ and by taking expectations on both sides we obtain

$$
e^{c_{3} t} E \Psi\left(X_{n}^{x}(t)\right)-\Psi\left(X_{n}^{x}(0)\right)=E \int_{0}^{t} e^{c_{3} s}\left(c_{3} \Psi\left(X_{n}^{x}(s)\right)+\mathcal{L}_{n} \Psi\left(X_{n}^{x}(s)\right)\right) d s .
$$

Now from condition (ii) it follows

$$
c_{3} \Psi\left(X_{n}^{x}(s)\right)+\mathcal{L}_{n} \Psi\left(X_{n}^{x}(s)\right) \leq-\mathcal{L} \Psi\left(X_{n}^{x}(s)\right)+k_{3}+\mathcal{L}_{n} \Psi\left(X_{n}^{x}(s)\right) .
$$

$$
\begin{aligned}
\Rightarrow e^{c_{3} t} E \Psi\left(X_{n}^{x}(t)\right)-\Psi\left(X_{n}^{x}(0)\right) & \leq E \int_{0}^{t} e^{c_{3} s}\left(-\mathcal{L} \Psi\left(X_{n}^{x}(s)\right)+k_{3}+\mathcal{L}_{n} \Psi\left(X_{n}^{x}(s)\right)\right) d s \\
& =E \int_{0}^{t} e^{c_{3} s}\left(-\mathcal{L} \Psi\left(X_{n}^{x}(s)\right)+\mathcal{L}_{n} \Psi\left(X_{n}^{x}(s)\right)\right) d s+\int_{0}^{t} e^{c_{3} s} k_{3} d s .
\end{aligned}
$$

Due to Corollary 2 we obtain

$$
E \int_{0}^{t} e^{c_{3} s}\left(-\mathcal{L} \Psi\left(X_{n}^{x}(s)\right)+\mathcal{L}_{n} \Psi\left(X_{n}^{x}(s)\right)\right) d s \rightarrow 0
$$


Therefore as $n \rightarrow \infty$, using the continuity of $\Psi$ and Lebesgue dominated convergence Theorem, we get

$$
\begin{aligned}
& e^{c_{3} t} E \Psi\left(X^{x}(t)\right) \leq \Psi(x)+\int_{0}^{t} e^{c_{3} s} k_{3} d s \\
&=\Psi(x)+\frac{k_{3}}{c_{3}}\left(e^{c_{3} t}-1\right) . \\
& \Rightarrow E \Psi\left(X^{x}(t)\right) \leq e^{-c_{3} t} \Psi(x)+\frac{k_{3}}{c_{3}}\left(1-e^{-c_{3} t}\right) .
\end{aligned}
$$

Now from condition (i) we get that for all $x \in H$

$$
\begin{gathered}
c_{1} E\left\|X^{x}(t)\right\|_{H}^{2}-k_{1} \leq E \Psi\left(X^{x}(t)\right) \leq e^{-c_{3} t}\left(c_{2}\|x\|_{H}^{2}-k_{2}\right)+\frac{k_{3}}{c_{3}}\left(1-e^{-c_{3} t}\right) \\
\leq c_{2} e^{-c_{3} t}\|x\|_{H}^{2}+\frac{k_{3}}{c_{3}}\left(1-e^{-c_{3} t}\right) . \\
\Rightarrow c_{1} E\left\|X^{x}(t)\right\|_{H}^{2} \leq c_{2} e^{-c_{3} t}\|x\|_{H}^{2}+\frac{k_{3}}{c_{3}}\left(1-e^{-c_{3} t}\right)+k_{1} \\
\Rightarrow E\left\|X^{x}(t)\right\|_{H}^{2} \leq \frac{c_{2}}{c_{1}} e^{-c_{3} t}\|x\|_{H}^{2}+\frac{1}{c_{1}}\left(k_{1}+\frac{k_{3}}{c_{3}}\right)
\end{gathered}
$$

By using the density of $\mathcal{D}(A)$ in $H$ and inequality (29) we conclude that by choosing $c=\frac{c_{2}}{c_{1}}, \beta=c_{3}$ and $M=\frac{1}{c_{1}}\left(k_{1}+\frac{k_{3}}{c_{3}}\right)$ inequality (30) holds for all $x \in H$, and hence the mild solution $\left\{X^{x}(t), t \geq 0\right\}$ of (11) is exponentially ultimate bounded in the m.s.s..

3.5. An "Ichikawa-type" Itô formula for mild solutions. In this subsection we prove that the Ichikawa type Itô formula for the mild solutions $\left\{X^{\xi}(t), t \geq 0\right\}$ obtained by Ichikawa for SPDEs driven by Gaussian noise in [15], can also be generalized to the case of the SPDE defined in (1). The advantage of introducing this "Ichikawa -type" Itô formula for mild solutions of (11) is that in this type of Itô formula ([36) ) below) there is no Yosida approximant appearing, unlike than in the Itô formula (15) obtained in Theorem 4. The disadvantage is however that compared to Theorem 4 we have to restrict the set of functions $\Psi:[0, T] \times H \rightarrow \mathbb{R}$ for which we can apply the Itô formula To this aim we introduce the following notation:

Let $\tilde{C}^{1,2}([0, T] \times H)\left(\right.$ resp. $\left.\quad \tilde{C}_{b}^{1,2}([0, T] \times H)\right)$ be the class of functions $\Psi \in$ $C^{1,2}([0, T] \times H)\left(\right.$ resp. $\left.\Psi \in C_{b}^{1,2}([0, T] \times H)\right)$ with the properties:

(I1) The function $\mathcal{L} \Psi(s, x)$ can be extended to a continuous function $\overline{\mathcal{L} \Psi}(s, x)$ on $[0, T] \times H$ for $x \in H$.

(I2) $\|\overline{\mathcal{L} \Psi}(s, x)\| \leq k\left(1+\|x\|^{2}\right)$, for $x \in H, s \in[0, T]$ and for some $k>0$.

Theorem 7. Let $\{S(t), t \geq 0\}$ be a pseudo-contraction semigroup generated by $A$ satisfying assumption (A1). Suppose assumption (A2) holds. Let the coefficients $F, B, f$ satisfy assumption (A3). Let $\left\{X^{\xi}(t), t \in[0, T]\right\}$ be the mild solution of (1) 
in $[0, T]$ with initial condition $\xi \in \mathcal{D}(A)$. For any $\Psi \in \tilde{C}^{1,2}([0, T] \times H)$ such that (17), (8), (9) are satisfied, the following Ichikawa type Itô Formula for mild solutions hold $P$-a.s. for all $t \in[0, T]$

$$
\begin{aligned}
& \Psi\left(t, X^{\xi}(t)\right)-\Psi(0, \xi)=\int_{0}^{t}\left(\Psi_{s}\left(s, X^{\xi}(s)\right)+\overline{\mathcal{L} \Psi}\left(s, X^{\xi}(s)\right)\right) d s \\
& +\int_{0}^{t}\left\langle\Psi_{x}\left(s, X^{\xi}(s)\right), B\left(X^{\xi}(s)\right) d W_{s}\right\rangle_{H} \\
& +\int_{0}^{t} \int_{H \backslash\{0\}}\left[\Psi\left(s, X^{\xi}(s)+f\left(v, X^{\xi}(s)\right)\right)-\Psi\left(s, X^{\xi}(s)\right)\right] q(d v, d s) .
\end{aligned}
$$

Proof. Here we assumed that $\Psi \in \tilde{C}^{1,2}([0, T] \times H)$. So, it satisfies the conditions (I1) and (I2). From (I1) the continuous extension of $\mathcal{L} \Psi(s, x)$ exists in $[0, T] \times H$, which is $\overline{\mathcal{L} \Psi}(s, x)$. It follows that if we denote again with $\left\{X_{n}(t)^{\xi}, t \geq 0\right\}$ the Yosida approximation of $\left\{X^{\xi}(t), t \geq 0\right\}$, then

$$
\begin{aligned}
& \overline{\mathcal{L} \Psi}\left(s, X^{\xi}(s)\right)=\lim _{n \rightarrow \infty} \mathcal{L} \Psi\left(s, X_{n}^{\xi}(s)\right) \\
& =\left\langle\Psi_{x}\left(s, X^{\xi}(s)\right), F\left(X^{\xi}(s)\right)\right\rangle_{H}+\lim _{n \rightarrow \infty}\left\langle\Psi_{x}\left(s, X_{n}^{\xi}(s)\right), A X_{n}^{\xi}(s)\right\rangle_{H} \\
& +\frac{1}{2} \operatorname{tr}\left(\Psi_{x x}\left(s, X^{\xi}(s)\right)\left(B\left(X^{\xi}(s)\right)\right) Q\left(B\left(X^{\xi}(s)\right)\right)^{*}\right) \\
& +\int_{H \backslash\{0\}}\left[\Psi\left(s, X^{\xi}(s)+f\left(v, X^{\xi}(s)\right)\right)-\Psi\left(s, X^{\xi}(s)\right)-\left\langle\Psi_{x}\left(s, X^{\xi}(s)\right), f\left(v, X^{\xi}(s)\right)\right\rangle_{H}\right] \beta(d v)
\end{aligned}
$$

where $X_{n}^{\xi}(s) \in \mathcal{D}(A)$. We know here that the $\operatorname{limit}_{n \rightarrow \infty}\left\langle\Psi_{x}\left(s, X_{n}^{\xi}(s)\right), A X_{n}^{\xi}(s)\right\rangle_{H}$ is well defined, as we assume (I1) and all other terms converge, as shown in the proof of Theorem [4 By (I2) $\overline{\mathcal{L} \Psi}\left(s, X^{\xi}(s)\right)$ is bounded by integrable function, so by applying Lebesgue dominated convergence theorem, we conclude that $\int_{0}^{t} \mathcal{L} \Psi\left(s, X_{n}^{\xi}(s)\right) d s \rightarrow \int_{0}^{t} \overline{\mathcal{L} \Psi}\left(s, X^{\xi}(s)\right) d s$, as $n \rightarrow \infty$. Hence we conclude that the Ichikawa type Itô Formula of (36) holds.

The proof of following Corollary is straight by using Remark 3 .

Corollary 5. Let $\{S(t), t \geq 0\}$ be a pseudo-contraction semigroup generated by $A$ satisfying assumption (A1). Suppose assumption (A2) holds. Let the coefficients $F, B, f$ satisfy assumption (A3). Let $\left\{X^{\xi}(t), t \in[0, T]\right\}$ be the mild solution of (11) in $[0, T]$ with initial condition $\xi \in \mathcal{D}(A)$. Then for all $\Psi \in \tilde{C}_{b}^{1,2}([0, T] \times H)$ the Ichikawa -type Itô formula (36) holds.

Remark 6. Whenever $\Psi \in \tilde{C}^{1,2}([0, T] \times H)\left(\Psi \in \tilde{C}_{b}^{1,2}([0, T] \times H)\right)$ i.e. $\overline{\mathcal{L}} \Psi(s, x)$ exists and the conditions of Theorem change the limit with the integral in the Ito formula for mild solutions of Theorem 4. Then the Itô formula for mild solutions of Theorem 4 (eq. (15) can be rewritten 
as follows-

$$
\begin{aligned}
& \int_{0}^{t} \lim _{n \rightarrow \infty}\left\langle\Psi_{x}\left(s, X_{n}^{\xi}(s)\right), A X_{n}^{\xi}(s)\right\rangle_{H} d s \\
& =\Psi\left(t, X^{\xi}(t)\right)-\Psi(0, \xi)-\int_{0}^{t}\left(\Psi_{s}\left(s, X^{\xi}(s)\right)\right) d s-\int_{0}^{t}\left\langle\Psi_{x}\left(s, X^{\xi}(s)\right), F\left(X^{\xi}(s)\right)\right\rangle_{H} d s \\
& -\int_{0}^{t} \frac{1}{2} \operatorname{tr}\left(\Psi_{x x}\left(s, X^{\xi}(s)\right)\left(B\left(X^{\xi}(s)\right)\right) Q\left(B\left(X^{\xi}(s)\right)\right)^{*}\right) d s \\
& -\int_{0}^{t} \int_{H \backslash\{0\}}\left[\Psi\left(s, X^{\xi}(s)+f\left(v, X^{\xi}(s)\right)\right)-\Psi\left(s, X^{\xi}(s)\right)-\left\langle\Psi_{x}\left(s, X^{\xi}(s)\right), f\left(v, X^{\xi}(s)\right)\right\rangle_{H}\right] \beta(d v) d s \\
& -\int_{0}^{t}\left\langle\Psi_{x}\left(s, X^{\xi}(s)\right), B\left(X^{\xi}(s)\right) d W_{s}\right\rangle_{H} \\
& -\int_{0}^{t} \int_{H \backslash\{0\}}\left[\Psi\left(s, X^{\xi}(s)+f\left(v, X^{\xi}(s)\right)\right)-\Psi\left(s, X^{\xi}(s)\right)\right] q(d v, d s) .
\end{aligned}
$$

Here we show through two examples how the Itô formula (15) in Theorem 4 obtained through Yosida approximation, can be related to the "Ichikawa type" -Itô formula (36) in Theorem 7

\section{Example 2:-}

Let $A$ be a symmetric linear operator and the assumptions of Theorem 4 hold. Assume that, for a fixed $l>0, l \in(0, \infty), \Psi(s, x)=e^{l A} \Lambda(s, x)$ and $\Lambda_{x}(s, x) \in \mathcal{D}(A)$. Also assume that $\Psi \in C^{1,2}\left(\mathbb{R}_{+} \times H\right)$ and satisfies the following property,

(i) There exists constants $c_{1}>0$ s.t. $\left\|\Psi_{x}(s, x)\right\| \leq c_{2}\|x\|_{H}$; for all $x \in H$.

Moreover assume (7), (8), (9) are satisfied.

$$
\left\langle\Psi_{x}\left(s, X_{n}^{\xi}(s)\right), A X_{n}^{\xi}(s)\right\rangle_{H} \leq \mathcal{C}\left\|X_{n}^{\xi}(s)\right\|_{H}^{2}
$$

Here,

$$
\begin{aligned}
& \left\langle\Psi_{x}\left(s, X_{n}^{\xi}(s)\right), A X_{n}^{\xi}(s)\right\rangle_{H} \\
& =\left\langle e^{l A} \Lambda_{x}\left(s, X_{n}^{\xi}(s)\right), A X_{n}^{\xi}(s)\right\rangle_{H} \\
& =\left\langle A^{*} e^{l A} \Lambda_{x}\left(s, X_{n}^{\xi}(s)\right), X_{n}^{\xi}(s)\right\rangle_{H} \\
& \leq \mathcal{C}\left\|X_{n}^{\xi}(s)\right\|_{H}^{2} .
\end{aligned}
$$

We can write from second to third equality, due to the fact that, $e^{l A} \Lambda_{x}\left(s, X_{n}^{\xi}(s)\right) \in$ $\mathcal{D}(A) \subset \mathcal{D}\left(A^{*}\right)$ as we assumed $\Lambda_{x}(s, x) \in \mathcal{D}(A)$ and $A$ to be a symmetric linear operator.

By Theorem 3 and Generalized Lebesgue Dominated Convergence theorem (Theorem 3.4 of [13]) we conclude that,

$$
\int_{0}^{t}\left\langle e^{l A} \Lambda_{x}\left(s, X_{n}^{\xi}(s)\right), A X_{n}^{\xi}(s)\right\rangle_{H} d s \rightarrow \int_{0}^{t}\left\langle A^{*} e^{l A} \Lambda_{x}\left(s, X^{\xi}(s)\right), X^{\xi}(s)\right\rangle_{H} d s
$$


$P$-a.s.. Therefore we can write the Itô formula for mild solutions of Theorem 4 for the function $\Psi(s, x)=e^{l A} \Lambda(s, x)$, for $x \in H$, as follows

$$
\begin{aligned}
& e^{l A} \Lambda\left(t, X^{\xi}(t)\right)-e^{l A} \Lambda(0, \xi)=\int_{0}^{t} e^{l A} \Lambda_{s}\left(s, X^{\xi}(s)\right) d s \\
& +\int_{0}^{t}\left\langle e^{l A} \Lambda_{x}\left(s, X^{\xi}(s)\right), F\left(X^{\xi}(s)\right)\right\rangle_{H} d s+\int_{0}^{t}\left\langle A^{*} e^{l A} \Lambda_{x}\left(s, X^{\xi}(s)\right), X^{\xi}(s)\right\rangle_{H} d s \\
& +\int_{0}^{t} \frac{1}{2} \operatorname{tr}\left(e^{l A} \Lambda_{x x}\left(s, X^{\xi}(s)\right)\left(B\left(X^{\xi}(s)\right)\right) Q\left(B\left(X^{\xi}(s)\right)\right)^{*}\right) d s \\
& +\int_{0}^{t} \int_{H \backslash\{0\}} e^{l A}\left[\Lambda\left(s, X^{\xi}(s)+f\left(v, X^{\xi}(s)\right)\right)-\Lambda\left(s, X^{\xi}(s)\right)-\left\langle\Lambda_{x}\left(s, X^{\xi}(s)\right), f\left(v, X^{\xi}(s)\right)\right\rangle_{H}\right] \beta(d v) d s \\
& +\int_{0}^{t}\left\langle e^{l A} \Lambda_{x}\left(s, X^{\xi}(s)\right), B\left(X^{\xi}(s)\right) d W_{s}\right\rangle_{H} \\
& +\int_{0}^{t} \int_{H \backslash\{0\}} e^{l A}\left[\Lambda\left(s, X^{\xi}(s)+f\left(v, X^{\xi}(s)\right)\right)-\Lambda\left(s, X^{\xi}(s)\right)\right] q(d v, d s)
\end{aligned}
$$

$P$-a.s. and by Theorem 7 we obtain

$$
\begin{aligned}
& \overline{\mathcal{L} \Psi}\left(s, X^{\xi}(s)\right)=\left\langle e^{l A} \Lambda_{x}\left(s, X^{\xi}(s)\right), F\left(X^{\xi}(s)\right)\right\rangle_{H}+\left\langle A^{*} e^{l A} \Lambda_{x}\left(s, X^{\xi}(s)\right), X^{\xi}(s)\right\rangle_{H} \\
& +\frac{1}{2} \operatorname{tr}\left(e^{l A} \Lambda_{x x}\left(s, X^{\xi}(s)\right)\left(B\left(X^{\xi}(s)\right)\right) Q\left(B\left(X^{\xi}(s)\right)\right)^{*}\right) \\
& +\int_{H \backslash\{0\}} e^{l A}\left[\Lambda\left(s, X^{\xi}(s)+f\left(v, X^{\xi}(s)\right)\right)-\Lambda\left(s, X^{\xi}(s)\right)-\left\langle\Lambda_{x}\left(s, X^{\xi}(s)\right), f\left(v, X^{\xi}(s)\right)\right\rangle_{H}\right] \beta(d v) .
\end{aligned}
$$

\section{Example 3:-}

Let $A$ be a symmetric linear operator. Now, we consider $\Psi(s, x)=e^{(t-s) A} \Gamma(x)$ for $x \in H$. Assume that $\Psi \in C^{1,2}\left(\mathbb{R}_{+} \times H\right)$ satisfies the condition (i) of Example 2 . Also assume that $\Gamma, \Gamma_{x} \in \mathcal{D}(A)$. Moreover assume (77), (8), (9) are satisfied.

$$
\Psi_{s}\left(s, X_{n}^{\xi}(s)\right)=\left(-A e^{(t-s) A} \Gamma\left(X_{n}^{\xi}(s)\right)\right) \rightarrow\left(-A e^{(t-s) A} \Gamma\left(X^{\xi}(s)\right)\right)
$$

$P$-a.s.. The convergence of all other terms are similar as in Example 2. So, we can write the Itô formula of Theorem 4 as, 
$(40)$

$$
\begin{aligned}
& \Gamma\left(X^{\xi}(t)\right)-e^{t A} \Gamma(\xi)=\int_{0}^{t}\left(-A e^{(t-s) A} \Gamma\left(X^{\xi}(s)\right)\right) d s \\
& +\int_{0}^{t}\left\langle e^{(t-s) A} \Gamma_{x}\left(X^{\xi}(s)\right), F\left(X^{\xi}(s)\right)\right\rangle_{H} d s+\int_{0}^{t}\left\langle A^{*} e^{(t-s) A} \Gamma_{x}\left(X^{\xi}(s)\right), X^{\xi}(s)\right\rangle_{H} d s \\
& +\int_{0}^{t} \frac{1}{2} \operatorname{tr}\left(e^{(t-s) A} \Gamma_{x x}\left(X^{\xi}(s)\right)\left(B\left(X^{\xi}(s)\right)\right) Q\left(B\left(X^{\xi}(s)\right)\right)^{*}\right) d s \\
& +\int_{0}^{t} \int_{H \backslash\{0\}}\left[e^{(t-s) A} \Gamma\left(X^{\xi}(s)+f\left(v, X^{\xi}(s)\right)\right)-e^{(t-s) A} \Gamma\left(X^{\xi}(s)\right)\right. \\
& \left.\quad-\left\langle e^{(t-s) A} \Gamma_{x}\left(X^{\xi}(s)\right), f\left(v, X^{\xi}(s)\right)\right\rangle_{H}\right] \beta(d v) d s \\
& +\int_{0}^{t}\left\langle e^{(t-s) A} \Gamma_{x}\left(X^{\xi}(s)\right), B\left(X^{\xi}(s)\right) d W_{s}\right\rangle_{H} \\
& +\int_{0}^{t} \int_{H \backslash\{0\}}\left[e^{(t-s) A} \Gamma\left(X^{\xi}(s)+f\left(v, X^{\xi}(s)\right)\right)-e^{(t-s) A} \Gamma\left(X^{\xi}(s)\right)\right] q(d v, d s)
\end{aligned}
$$

$P$-a.s. and by Theorem [7, we obtain

$$
\begin{aligned}
& \overline{\mathcal{L} \Psi}\left(s, X^{\xi}(s)\right)=\left\langle e^{(t-s) A} \Gamma_{x}\left(X^{\xi}(s)\right), F\left(X^{\xi}(s)\right)\right\rangle_{H}+\left\langle A^{*} e^{(t-s) A} \Gamma_{x}\left(X^{\xi}(s)\right), X^{\xi}(s)\right\rangle_{H} \\
& +\frac{1}{2} \operatorname{tr}\left(e^{(t-s) A} \Gamma_{x x}\left(X^{\xi}(s)\right)\left(B\left(X^{\xi}(s)\right)\right) Q\left(B\left(X^{\xi}(s)\right)\right)^{*}\right) \\
& +\int_{H \backslash\{0\}}\left[e^{(t-s) A} \Gamma\left(X^{\xi}(s)+f\left(v, X^{\xi}(s)\right)\right)-e^{(t-s) A} \Gamma\left(X^{\xi}(s)\right)\right. \\
& \left.-\left\langle e^{(t-s) A} \Gamma_{x}\left(X^{\xi}(s)\right), f\left(v, X^{\xi}(s)\right)\right\rangle_{H}\right] \beta(d v) .
\end{aligned}
$$

\section{An Itô Formula type InEQUALity}

We assume in the whole section that $\{S(t), t \geq 0\}$ is a pseudo-contraction semigroup generated by $A$ satisfying assumption (A1), that (A2) holds. and that the coefficients $F, B, f$ satisfy assumption (A3). Let $X^{x}(t):==X(t, 0 ; x) \in H$ denote the the mild solution of (5) with deterministic initial condition $x \in H . X^{x}(t)$ is a homogeneous Markov process (see, section 3.4 of [13] and section 6 of [1]), section 5.4 [24].

Let $\Psi \in C_{b}^{2}(H)$ and $x \in \mathcal{D}(A)$, then the the operator $\mathcal{L}$ defined in (11) is well defined in all its terms and acts on $\Psi$ as follows: .

$$
\begin{aligned}
\mathcal{L} \Psi(x):= & \left\langle\Psi_{x}(x), A x+F(x)\right\rangle_{H}+\frac{1}{2} \operatorname{tr}\left(\Psi_{x x}(x)(B(x)) Q(B(x))^{*}\right) \\
& +\int_{H \backslash\{0\}}\left[\Psi(x+f(v, x))-\Psi(x)-\left\langle\Psi_{x}(x), f(v, x)\right\rangle_{H}\right] \beta(d v) .
\end{aligned}
$$

Let $P_{t}$ be the semigroup acting on $\Psi \in C_{b}^{2}(H)$, such that

$$
\left[P_{t} \Psi\right](x)=E\left[\Psi\left(X^{x}(t)\right)\right] \quad \text { for } x \in H
$$


Theorem 8. Assume that the solution $X^{x}(t)$ of SPDE (11) with initial condition $x \in \mathcal{D}(A)$ satisfies $X^{x}(t) \in \mathcal{D}(A)$ for all $t \geq 0$. Then for $\Psi \in C_{b}^{2}(H)$

$$
\left[P_{t} \Psi\right](x)-\Psi(x)=\int_{0}^{t}\left[P_{s} \mathcal{L} \Psi\right](x) d s
$$

and

$$
\lim _{t \downarrow 0} \frac{\left[P_{t} \Psi\right](x)-\Psi(x)}{t}=[\mathcal{L} \Psi](x) .
$$

Proof. We refer sections 4.1, 4.2 of [10] or sections 3.2, 3.3 of [3] for related theory.

$$
\begin{gathered}
\Psi\left(X^{x}(t)\right)-\Psi(x)=\int_{0}^{t} \mathcal{L} \Psi\left(X^{x}(s)\right) d s \\
+\int_{0}^{t}\left\langle\Psi_{x}\left(X^{x}(s)\right), B\left(X^{x}(s)\right) d W_{s}\right\rangle_{H} \\
+\int_{0}^{t} \int_{H \backslash\{0\}}\left[\Psi\left(X^{x}(s)+f\left(v, X^{x}(s)\right)\right)-\Psi\left(X^{x}(s)\right)\right] q(d v, d s) .
\end{gathered}
$$

Now take the expectation on both sides of (43). Second and third term of R.H.S. of (43) will be zero, because of martingale and we get,

$$
E\left[\Psi\left(X^{x}(t)\right)\right]-E[\Psi(x)]=\int_{0}^{t} E\left[\mathcal{L} \Psi\left(X^{x}(s)\right)\right] d s .
$$

Then we substitute $\left[P_{t} \Psi\right](x)=E\left[\Psi\left(X^{x}(t)\right)\right]$, and we get

$$
\left[P_{t} \Psi\right](x)-\Psi(x)=\int_{0}^{t}\left[P_{s} \mathcal{L} \Psi\right](x) d s .
$$

Again to prove (42), we can rewrite (43) as,

$$
\begin{gathered}
d \Psi\left(X^{x}(t)\right)=\left\langle\frac{d \Psi\left(X^{x}(t)\right)}{d x}, A X^{x}(t)+F\left(X^{x}(t)\right)\right\rangle_{H} d t \\
+\frac{1}{2} \operatorname{tr}\left(\frac{d^{2} \Psi\left(X^{x}(t)\right)}{d x^{2}}\left(B\left(X^{x}(t)\right) Q^{1 / 2}\right)\left(B\left(X^{x}(t)\right) Q^{1 / 2}\right)^{*}\right) d t \\
+\int_{H \backslash\{0\}}\left[\Psi\left(X^{x}(t)+f\left(v, X^{x}(t)\right)\right)-\Psi\left(X^{x}(t)\right)-\left\langle\frac{d \Psi\left(X^{x}(t)\right)}{d x}, f\left(v, X^{x}(t)\right)\right\rangle_{H}\right] \beta(d v) d t \\
+\left\langle\frac{d \Psi\left(X^{x}(t)\right)}{d x}, B\left(X^{x}(t)\right) d W_{t}\right\rangle_{H} \\
+\int_{H \backslash\{0\}}\left[\Psi\left(X^{x}(t)+f\left(v, X^{x}(t)\right)\right)-\Psi\left(X^{x}(t)\right)\right] q(d v, d t) .
\end{gathered}
$$

Now, since $\left[P_{t} \Psi\right](x)=E\left[\Psi\left(X^{x}(t)\right)\right]$, so by Lebesgue dominated convergence theorem

$$
\begin{gathered}
\lim _{t \downarrow 0} \frac{\left[P_{t} \Psi\right](x)-\Psi(x)}{t} \\
=E \lim _{t \downarrow 0} \frac{1}{t} \int_{0}^{t}\left\langle\frac{d \Psi\left(X^{x}(s)\right)}{d x}, A X^{x}(s)+F\left(X^{x}(s)\right)\right\rangle_{H} d s
\end{gathered}
$$




$$
\begin{aligned}
&+ \frac{1}{2} E \lim _{t \downarrow 0} \frac{1}{t} \int_{0}^{t} \operatorname{tr}\left(\frac{d^{2} \Psi\left(X^{x}(s)\right)}{d x^{2}}\left(B\left(X^{x}(s)\right) Q^{1 / 2}\right)\left(B\left(X^{x}(s)\right) Q^{1 / 2}\right)^{*}\right) d s \\
&+E \lim _{t \downarrow 0} \frac{1}{t} \int_{0}^{t} \int_{H \backslash\{0\}}\left[\Psi\left(X^{x}(s)+f\left(v, X^{x}(s)\right)\right)-\Psi\left(X^{x}(s)\right)-\left\langle\frac{d \Psi\left(X^{x}(s)\right)}{d x}, f\left(v, X^{x}(s)\right)\right\rangle_{H}\right] \beta(d v) d s \\
&=\left\langle\frac{d \Psi(x)}{d x}, A x+F(x)\right\rangle_{H}+\frac{1}{2} \operatorname{tr}\left(\frac{d^{2} \Psi(x)}{d x^{2}}\left(B(x) Q^{1 / 2}\right)\left(B(x) Q^{1 / 2}\right)^{*}\right) \\
&+\int_{H \backslash\{0\}}\left[\Psi(x+f(v, x))-\Psi(x)-\left\langle\frac{d \Psi(x)}{d x}, f(v, x)\right\rangle_{H}\right] \beta(d v) \\
&=[\mathcal{L} \Psi](x) .
\end{aligned}
$$

Hence the proof follows.

Now we consider also the case when $X^{x}(t) \notin \mathcal{D}(A)$. $\Psi$ is independent of time $t$ and $\Psi \in \tilde{C}_{b}^{2}(H)$, where $\tilde{C}_{b}^{2}(H)$ is defined like $\tilde{C}_{b}^{1,2}(H)$ however closing the operator $\mathcal{L}$ over functions defined on $C_{b}^{2}(H)$, i.e. $\tilde{C}_{b}^{2}(H)$ is the class of functions $\Psi \in C_{b}^{2}(H)$ satisfying the properties:

(1) The function $\mathcal{L} \Psi(x)$ can be extended to a continuous function $\overline{\mathcal{L} \Psi}(x)$ on $H$ for $x \in H$.

$(\tilde{2})\|\overline{\mathcal{L} \Psi}(x)\| \leq k\left(1+\|x\|^{2}\right)$, for $x \in H$ and for some $k>0$.

Corollary 6. Assume that $\overline{\mathcal{L} \Psi}$ satisfies the condition

$$
\|\overline{\mathcal{L} \Psi}(y)-\overline{\mathcal{L} \Psi}(z)\| \leq k\|y-z\|^{2}\left(\|y\|^{2}+\|z\|^{2}\right)
$$

for some $k>0$. Then

$$
\left[P_{t} \Psi\right](x)-\Psi(x)=\int_{0}^{t}\left[P_{s} \overline{\mathcal{L} \Psi}\right](x) d s, \quad \text { for } x \in \mathcal{D}(A)
$$

and

$$
\lim _{t \downarrow 0} \frac{\left[P_{t} \Psi\right](x)-\Psi(x)}{t}=[\overline{\mathcal{L} \Psi}](x), \quad \text { for } x \in \mathcal{D}(A) .
$$

Proof. For the proof we refer to A. Ichikawa [15]. It can be shortly described as follows: to prove (45), we take the expectation on both sides of (36) and use the relation $\left[P_{t} \Psi\right](x)=E\left[\Psi\left(X^{x}(t)\right)\right]$. The condition (44) assures the continuity of $P_{s} \overline{\mathcal{L} \Psi}$ in $s$. Therefore we can write

$$
\lim _{t \downarrow 0} \frac{\left[P_{t} \Psi\right](x)-\Psi(x)}{t}=E \lim _{t \downarrow 0} \frac{1}{t} \int_{0}^{t} \overline{\mathcal{L} \Psi}\left(X^{x}(s)\right) d s=[\overline{\mathcal{L} \Psi}](x) .
$$

Hence the proof.

We define

$$
[\mathcal{A} \Psi](x):=[\overline{\mathcal{L} \Psi}](x), \quad \text { for } x \in \mathcal{D}(A), \Psi \in \tilde{C}_{b}^{2}(H) .
$$

We call $\mathcal{A}$ the "weak generator of the Markov process $X^{x}(t)$ ".

The existence of $\overline{\mathcal{L} \Psi}$ is rather restrictive. Therefore we introduce a class of functions which is larger than $\tilde{C}_{b}^{2}(H)$, where continuous extension of the function $\mathcal{L} \Psi$ is not required. 
Lemma 4. Let $\Psi(x) \in C^{2}(H)$ satisfy the property:

(i) The function $\mathcal{L} \Psi(x) \leq \mathcal{U}(x)$, for $x \in \mathcal{D}(A)$, where $\mathcal{U}(x)$ is a continuous function on $H$ such that $\|\mathcal{U}(x)\| \leq k\left(1+\|x\|^{2}\right)$ for some $k>0$.

Moreover assume that conditions (7), (8), (9) hold.

Then

$\Psi\left(X^{x}(t)\right)-\Psi(x) \leq \int_{0}^{t} \mathcal{U}\left(X^{x}(s)\right) d s+\int_{0}^{t}\left\langle\Psi_{x}\left(X^{x}(s)\right), B\left(X^{x}(s)\right) d W_{s}\right\rangle_{H}$

$$
+\int_{0}^{t} \int_{H \backslash\{0\}}\left[\Psi\left(X^{x}(s)+f\left(v, X^{x}(s)\right)\right)-\Psi\left(X^{x}(s)\right)\right] q(d v, d s) .
$$

If, in particular, $\mathcal{U}(x)=0$, then $\Psi\left(X^{x}(t)\right)$ is a supermartingale.

Proof. $\int_{0}^{t} \mathcal{U}\left(X^{x}(s)\right) d s$ is well defined. By Corollary 2

$$
\int_{0}^{t} \lim _{n \rightarrow \infty} \mathcal{L}_{n} \Psi\left(X_{n}^{x}(s)\right) d s \leq \int_{0}^{t} \mathcal{U}\left(X^{x}(s)\right) d s .
$$

Therefore we conclude (48).

For the second part, we take the conditional expectation in both sides of (48), then we get

$$
E\left[\Psi\left(X^{x}(t)\right) \mid \mathcal{F}_{0}^{X}\right]-E\left[\Psi(x) \mid \mathcal{F}_{0}^{X}\right] \leq 0
$$

since $\mathcal{U}(x)=0$ and terms containing the Gaussian and non-Gaussian noise in the R.H.S. of (48) are martingales.

$$
\Rightarrow E\left[\Psi\left(X^{x}(t)\right) \mid \mathcal{F}_{0}^{X}\right] \leq \Psi(x) .
$$

Therefore a supermartingale.

Now, through an example, we show how Ichikawa type inequality (48) of Lemma 4 might be used to prove that a mild solution is exponentially stable in the mean square sense.

\section{Example 4:-}

Consider the stochastic heat equation

$$
d X(x, t)=\frac{\partial^{2}}{\partial x^{2}} X(x, t) d t+B(X(x, t)) d W_{t}+\int_{H \backslash\{0\}} f(v) X(x, t) q(d v, d t),
$$

with

$$
\begin{gathered}
0<x<1 . \\
X(0, t)=X(1, t)=0 ; \quad X(x, 0)=X_{0}(x) ; \quad X_{0}, B, f \in L_{2}(0,1) .
\end{gathered}
$$

Here we take $H=L_{2}(0,1), A=d^{2} / d x^{2}$ and

$$
\mathcal{D}(A)=\left\{f \in H \mid f^{\prime}, f^{\prime \prime} \in H ; f(0)=f(1)=0\right\} .
$$

Since $A$ has eigenvectors $\{\sqrt{2} \sin n \pi x\}$ and eigenvalues $\left\{-n^{2} \pi^{2}\right\}$ for $n \in \mathbb{N}$. Then $X \in \mathcal{D}(A),\langle A X, X\rangle \leq-\pi^{2}\|X\|^{2}$ (see example 6.1 of [15]). 
Now consider the function $\Psi(x)=\|x\|^{2}$. Therefore $\Psi_{x}(x)=2 x$ and $\Psi_{x x}=2$. Hence, for $x \in \mathcal{D}(A)$

$$
\begin{gathered}
\left\langle\Psi_{x}(x), A x\right\rangle \leq-2 \pi^{2}\|x\|^{2}, \\
\frac{1}{2} \operatorname{tr}\left(\Psi_{x x}(x)(B(x)) Q(B(x))^{*}\right)=\operatorname{tr}\left((B(x)) Q(B(x))^{*}\right) \leq l\left(1+\|x\|^{2}\right),
\end{gathered}
$$

by (A3) and

$$
\begin{aligned}
& \int_{H \backslash\{0\}}\left[\Psi(x+f(v) x)-\Psi(x)-\left\langle\Psi_{x}(x), f(v) x\right\rangle\right] \beta(d v) \\
& \leq \int_{H \backslash\{0\}}\|f(v) x\|^{2} \times \sup _{0 \leq \theta \leq 1}\left\|\Psi_{x x}(x+\theta f(v) y)\right\| \beta(d v) \\
& \left.\leq 2 l\left(1+\|x\|^{2}\right) \quad \text { [since, } \Psi_{x x}=2 .\right]
\end{aligned}
$$

by (A3). To get the above inequality we followed the argument used in the proof of Theorem 4 Therefore for $x \in \mathcal{D}(A)$,

$$
\begin{aligned}
\mathcal{L} \Psi(x)=\mathcal{L}\|x\|^{2} & \leq-2 \pi^{2}\|x\|^{2}+(l+2 l)\left(1+\|x\|^{2}\right) \\
& =\left(-2 \pi^{2}+3 l\right)\|x\|^{2}+3 l
\end{aligned}
$$

So that $\Psi$ satisfies (i) in Lemma4,

$$
\begin{aligned}
\|X(t)\|^{2}-\|X(0)\|^{2} \leq & \int_{0}^{t}\left\{\left(-2 \pi^{2}+3 l\right)\|X(s)\|^{2}+3 l\right\} d s \\
& +\int_{0}^{t}\left\langle 2 X(s), B(X(s)) d W_{s}\right\rangle \\
& +\int_{0}^{t} \int_{H \backslash\{0\}}\left[\|X(s)+f(v) X(s)\|^{2}-\|X(s)\|^{2}\right] q(d v, d s) .
\end{aligned}
$$

So, from Lemma 4, we obtain the inequality of (50) for the function $\Psi(x)=\|x\|^{2}$.

Now whenever $\pi^{2}>\frac{3}{2} l$, then for some constant $k>0$

$$
\begin{aligned}
\|X(t)\|^{2}-\|X(0)\|^{2} \leq & \int_{0}^{t}\left\{-k\|X(s)\|^{2}+3 l\right\} d s+\int_{0}^{t}\left\langle 2 X(s), B(X(s)) d W_{s}\right\rangle \\
& +\int_{0}^{t} \int_{H \backslash\{0\}}\left[\|X(s)+f(v) X(s)\|^{2}-\|X(s)\|^{2}\right] q(d v, d s) .
\end{aligned}
$$


Now applying expectation on both sides of (51)

$$
\begin{aligned}
E\left[\|X(t)\|^{2}\right] & \leq-k \int_{0}^{t} E\left[\|X(s)\|^{2}\right] d s+3 l t+\|X(0)\|^{2} \\
& \left.\leq-k \int_{0}^{t} E\left[\|X(s)\|^{2}\right] d s+\lambda\|X(0)\|^{2} \quad \text { [for sufficiently large } \lambda>0\right] .
\end{aligned}
$$

Therefore, by Gronwall's lemma

$$
E\left[\|X(t)\|^{2}\right] \leq \lambda e^{-k t}\|X(0)\|^{2},
$$

hence by Definition [5 we conclude that the solution $X(t)$ is exponentially stable in the mean square sense.

\section{Mild Itô Formula Written IN terms of the SEMigroup}

In [6] an Itô formula written in terms of the semigroup $\{S(t)\}_{t \geq 0}$ of the Drift operator $A$ was derived for the Gaussian case. There the mild Itô process was transformed to a standard Itô process to which the standard Itô formula was applied. By relating this transformed standard Itô process with the original mild Itô process with a suitable relation, the mild Itô formula written in terms of $\{S(t)\}_{t \geq 0}$ was obtained.

We present here the method and result for the non Gaussian case

We consider the SPDE with values in $H$ as

$$
d X(t)=(A X(t)+F(X(t))) d t+\int_{H \backslash\{0\}} f(v, X(t)) q(d v, d t)
$$

with initial condition $X(0) \in H$. Where $H$ is a real separable Hilbert space, $A$ is the generator of $S(t)$ a pseudo-contraction semigroup $\{S(t)\}_{t \geq 0}$ and conditions (A1), (A3) are satisfied. (To simplify formulas we skip the Gaussian term ). Then from Definition 2, the mild solutions are defined as

$X(t)=S(t) X(0)+\int_{0}^{t} S(t-s) F(X(s)) d s+\int_{0}^{t} \int_{H \backslash\{0\}} S(t-s) f(v, X(s)) q(d v, d s)$

with probability one for $t \in[0, T]$.

Theorem 9. Assume that $\Psi \in C^{1,2}([0, T] \times H), \Psi:[0, T] \times H \rightarrow \mathbb{R}$. Also assume that the conditions (7), (8), (9) hold. Then the following mild Itô formula holds 
$(54)$

$$
\begin{aligned}
& \Psi(t, X(t))=\Psi(S(t) X(0))+\int_{0}^{t}\left(\partial_{1} \Psi\right)(s, S(t-s) X(s)) d s \\
& +\int_{0}^{t}\left(\partial_{2} \Psi\right)(s, S(t-s) X(s)) S(t-s) F(X(s)) d s \\
& +\int_{0}^{t} \int_{H \backslash\{0\}}[\Psi(s, S(t-s) X(s)+S(t-s) f(v, X(s)))-\Psi(s, S(t-s) X(s)) \\
& \left.\quad-\left\langle\left(\partial_{2} \Psi\right)(s, S(t-s) X(s)), S(t-s) f(v, X(s))\right\rangle\right] \beta(d v) d s \\
& +\int_{0}^{t} \int_{H \backslash\{0\}}[\Psi(s, S(t-s) X(s)+S(t-s) f(v, X(s)))-\Psi(s, S(t-s) X(s))] q(d v, d s) \\
& P-\text { a.s. for all } t \in[0, T] . \quad H e r e\left(\partial_{1} \Psi\right)(t, x)=\left(\frac{\partial \Psi}{\partial t}\right)(t, x) \text { and }\left(\partial_{2} \Psi\right)(t, x)= \\
& \left(\frac{\partial \Psi}{\partial x}\right)(t, x) . \quad\left(\partial_{1} \Psi\right) \in C([0, T] \times H, \mathbb{R}) \text { and }\left(\partial_{2} \Psi\right) \in C([0, T] \times H, \mathcal{L}(H, \mathbb{R})) .
\end{aligned}
$$

Proof. Here we use the transformation technique, given in [11. For existence and uniqueness of the mild solutions w.r.t. cPrm we refer [1].

We remark that the integrals in (54) are well defined due to the conditions (7), (8), (91). This can be proven in a similar way as in the Itô formula for SDEs proven in Theorem 3.7.2 in [18.

Let $U_{t} \in \mathcal{L}(H), t \in[0, \infty)$, is a strongly continuous pseudo-contractive semigroup on $H$ and $S(t-s)=U_{(t-s)} \in \mathcal{L}(H)$ for all $0 \leq s \leq t \leq T$.

Then there exists a separable $\mathbb{R}$-Hilbert space $\left(\mathcal{H},\langle., .\rangle_{\mathcal{H}},\|.\|_{\mathcal{H}}\right)$ with $H \subset \mathcal{H}$ and $\|v\|_{H}=\|v\|_{\mathcal{H}}$ for all $v \in H$ and a strongly continuous group $\mathcal{U}_{t} \in \mathcal{L}(\mathcal{H}), t \in \mathbb{R},($ Here we use the fact that, the strongly continuous pseudo-contractive semigroup can be dilated to strongly continuous group); such that

$$
U_{t}(v)=P\left(\mathcal{U}_{t}(v)\right)
$$

for all $v \in H \subset \mathcal{H}$ and all $t \in[0, \infty)$ where $P: \mathcal{H} \rightarrow H$ is the orthogonal projection from $\mathcal{H}$ to $H$.

Now we transform our mild Itô process $X:[0, T] \times \Omega \rightarrow H$ to a standard Itô process $\bar{X}$, by following the technique of [11, roughly speaking by multiplying the mild Itô process with $\mathcal{U}_{-t}$ for $t \in[0, T]$. Let, $\bar{X}:[0, T] \times \Omega \rightarrow \mathcal{H}$ be the unique adapted, càdlàg stochastic process such that,

$$
\bar{X}_{t}=X(0)+\int_{0}^{t} \mathcal{U}_{-s} F(X(s)) d s+\int_{0}^{t} \int_{\mathcal{H} \backslash\{0\}} \mathcal{U}_{-s} f(v, X(s)) q(d v, d s) .
$$

$P$-a.s. for all $t \in[0, T]$. Here we use the following transformation, 


$$
\begin{aligned}
P\left(\mathcal{U}_{t}\left(\bar{X}_{s}\right)\right)= & P\left(\mathcal{U}_{t}(X(0))\right)+\int_{0}^{s} P \mathcal{U}_{(t-u)} F(X(u)) d u+\int_{0}^{s} \int_{H \backslash\{0\}} P \mathcal{U}_{(t-u)} f(v, X(u)) q(d v, d u) \\
= & S(t) X(0)+\int_{0}^{s} S(t-u) F(X(u)) d u+\int_{0}^{s} \int_{H \backslash\{0\}} S(t-u) f(v, X(u)) q(d v, d u) \\
= & S(t-s)\left(S(s) X(0)+\int_{0}^{s} S(s-u) F(X(u)) d u+\int_{0}^{s} \int_{H \backslash\{0\}} S(s-u) f(v, X(u)) q(d v, d u)\right) \\
= & S(t-s) X(s) . \\
& \Rightarrow P\left(\mathcal{U}_{t}\left(\bar{X}_{t}\right)\right)=X(t) \text { and } P\left(\mathcal{U}_{t}\left(\bar{X}_{0}\right)\right)=S(t) X(0) .
\end{aligned}
$$

$P$-a.s. for all $s, t \in[0, T]$ with $s \leq t$.

Now we will apply the Itô formula for strong solutions of [19] to the test function $\Psi\left(s, P\left(\mathcal{U}_{t}(v)\right)\right)$ for $s \in[0, t], v \in \mathcal{H}$

$$
\begin{aligned}
& \Psi(t, X(t))=\Psi\left(t, P\left(\mathcal{U}_{t}\left(\bar{X}_{t}\right)\right)\right)=\Psi\left(P\left(\mathcal{U}_{t}\left(\bar{X}_{0}\right)\right)\right)+\int_{0}^{t}\left(\partial_{1} \Psi\right)\left(s, P\left(\mathcal{U}_{t}\left(\bar{X}_{s}\right)\right)\right) d s \\
& +\int_{0}^{t}\left(\partial_{2} \Psi\right)\left(s, P\left(\mathcal{U}_{t}\left(\bar{X}_{s}\right)\right)\right) P \mathcal{U}_{(t-s)} F(X(s)) d s \\
& +\int_{0}^{t} \int_{H \backslash\{0\}}\left[\Psi\left(s, P\left(\mathcal{U}_{t}\left(\bar{X}_{s}\right)\right)+P \mathcal{U}_{(t-s)} f(v, X(s))\right)-\Psi\left(s, P\left(\mathcal{U}_{t}\left(\bar{X}_{s}\right)\right)\right)\right. \\
& \left.\quad-\left\langle\left(\partial_{2} \Psi\right)\left(s, P\left(\mathcal{U}_{t}\left(\bar{X}_{s}\right)\right)\right), P \mathcal{U}_{(t-s)} f(v, X(s))\right\rangle\right] \beta(d v) d s \\
& +\int_{0}^{t} \int_{H \backslash\{0\}}\left[\Psi\left(s, P\left(\mathcal{U}_{t}\left(\bar{X}_{s}\right)\right)+P \mathcal{U}_{(t-s)} f(v, X(s))\right)-\Psi\left(s, P\left(\mathcal{U}_{t}\left(\bar{X}_{s}\right)\right)\right] q(d v, d s)\right.
\end{aligned}
$$

$P$-a.s. for all $\Psi \in C^{1,2}([0, T] \times H)$. Now substituting (55) and (57) in (58), we get our Mild Itô formula of (54).

Remark 7. The formula (40) for the function $\Psi(s, x)=e^{(t-s) A} \Gamma(x)$ was obtained through the Itô formula (15) in Theorem 4, as well as the "Ichikawa type" -Itô formula (36) in Theorem $\square$ and is obtained also applying the Itô formula (54) in Theoren 9

Acknowledgements: We are very grateful to Prof. P. Sundar (Louisiana State University) for useful discussions related to this work

\section{REFERENCES}

[1] Albeverio, S., Mandrekar, V. and Rüdiger, B. :Existence of mild solutions for SDE's and semilinear equations with non-Gaussian noise, Stochastic Proc. and Appl. 119(2009)835-863.

[2] Albeverio, S., Rüdiger, B. :Stochastic integrals and the Lévy-Ito decomposition theorem on separable Banach spaces, Stoch. Anal. Appl. 23 (2005), no. 2, 217-253.

[3] Applebaum, D. :Lévy Process and Stochastic Calculus (Second Edition), Cambridge University Press, (2009). 
[4] Brzeźniak, Z., Hausenblas, E., Zhu, J. :Maximal inequalities for Stochastic convolutions driven by Compensated Poisson Random Measures in Banach spaces, arXiv, (2010).

[5] Curtain, R. F. :Markov process generated by linear stochastic evolution equations, Stochastics, 5(1982), 135-165.

[6] Da Prato, G., Jentzen, A., Röckner, M. :A mild Itô formula for SPDEs, arXiv, (September 6,2011).

[7] Da Prato, G., Zabczyk, J. :Stochastic Equations in Infinite Dimensions, Cambridge University Press, (1992).

[8] Da Prato, G., Zabczyk, J. :Second Order Partial Differential Equations in Hilbert Spaces, Cambridge University Press, (2004).

[9] Engel, K.J. and Nagel, R. :One-Parameter Semigroups for Linear Evoulution Equations, Springer-Verlag New York, Inc.(2000).

[10] Ethier, S.N. and Kurtz, T.G. :Markov Processes, Characterization and Convergence, John Wiley and Sons, Inc.(1986).

[11] Fillipović, D., Tappe, S., Teichmann, J. :Term Structure Models Driven by Wiener Process and Poisson Measures: Existence and Positivity, SIAM J. Finan. Math. 1(1), 523-554, (2010).

[12] Fillipović, D., Tappe, S., Teichmann, J. :Jump-diffusions in Hilbert spaces: existence, stability and numerics, Stochastics 82, 5(2010), 475-520.

[13] Gawarecki, L. and Mandrekar, V. :Stochastic Differential Equation in Infinite Dimension, Springer Heidelberg Dordrecht London New York,(2011).

[14] Ichikawa, A. :Stability of semilinear stochastic evolution equations, J. Math. Anal. Appl. 90, 12-44(1982).

[15] Ichikawa, A. :Semilinear stochastic evolution equations: boundedness, stability and invariant measures, Stochastics 12, 1-39(1984).

[16] Ikeda, N., Watanabe, S. :Stochastic Differential Equations and Diffusion Processes, NorthHolland Mathematical Library (1989).

[17] Kallenberg, O. :Foundations of Modern Probability(Second Edition), by the Applied Probability Trust, 2002.

[18] Mandrekar, V. and Rüdiger, B.:Stochastic Integration in Banach Spaces, Springer Cham Heidelberg New York Dordrecht London,(2015).

[19] Mandrekar, V., Rüdiger, B., Tappe, S. :Ito's Formula for Banach Space valued Jump Processes driven by Poisson Random Measures, Seminar on Stochastic Analysis, Random Fields and Applications, Centro Stefano Franscini, Ascona (2011), Birkhäuser, May 2013.

[20] Mandrekar, V. and Wang, L. :Asymptotic Properties of SPDE in Hilbert Spaces driven by Non-Gaussian noise, Communications on Stochastic Analysis,Vol. 5,No. 2(2011) 309-331.

[21] Peszat, S., Zabczyk, J. :Stochastic Partial Differential Equations with Lévy Noise, Cambridge University Press (2007).

[22] Revuz, D., Yor, M. :Continuous Martingales and Brownian Motion, Springer-Verlag Berlin Heidelberg 1991, 1994, 1999.

[23] Rüdiger, B. : Stochastic integration with respect to Poisson random measure on seperable Banach space, Stoch. Stoch. Rep. 76(2004)213-242.

[24] Rüdiger, B., Ziglo, G. :Ito Formula for Stochastic Integrals w.r.t. Compensated Poisson Random Measures on Separable Banach Spaces, Stochastics 78 (2006), no. 6, 377-410.

[25] Shreve, Steven E. :Stochastic Calculus for Finance II, Springer-Verlag New York,LLC(2004).

[26] Skorokhod, A.V. :Studies in the Theory of Random Processes, Addison-Wesley, Reading, Massachusetts (1965).

[27] Sz.-Nagy, B. :Sur les contractions de l'espace de Hilbert, Acta Sci. Math. Szeged 15 (1953), $87-92$.

[28] Sz.-Nagy, B. :Transfotmations de l'espace de Hilbert, functions de type positif sur un groupe, Acta Sci. Math. Szeged 15 (1954), 104-114.

[29] Sz.-Nagy, B., Foias, C. :Harmonic analysis of operators on Hilbert space, Translated from the French and revised. North-Holland Publishing Co., Amsterdam, 1970. 
Sergio Albeverio: Institut für Angewandte Mathematik Abteilung Wahrscheinlichkeitstheorie, Rheinische Friedrich-Wilhelms-Universität Bonn, Endenicher Allee 60, 53115 Bonn, Germany

E-mail address: albeverio@uni-bonn.de / albeverio@yahoo.com

Leszek Gawarecki: Department of Mathematics Kettering University, 1700 University Ave Flint, MI 48504 USA

E-mail address: lgawarec@kettering.edu

Vidyadhar Mandrekar: Department of Statistics and Probability, Michigan State University, East Lansing, MI 48823 USA

E-mail address: atmah@stt.msu.edu / atma1m@gmail.com

Barbara Rüdiger: Bergische Universität Wuppertal, Fakultät für Mathematik und Naturwissenschaften, Gaussstrasse 20, 42119 Wuppertal, Germany

E-mail address: ruediger@uni-wuppertal.de

Barun Sarkar: Bergische Universität Wuppertal, Fakultät für Mathematik und Naturwissenschaften, Gaussstrasse 20, 42119 Wuppertal, Germany

E-mail address: barunsarkar.math@gmail.com 